NBER WORKING PAPER SERIES

\title{
IDENTIFICATION AND ESTIMATION OF GAUSSIAN AFFINE TERM STRUCTURE MODELS
}

\author{
James D. Hamilton \\ Jing Cynthia Wu \\ Working Paper 17772 \\ http://www.nber.org/papers/w17772 \\ NATIONAL BUREAU OF ECONOMIC RESEARCH \\ 1050 Massachusetts Avenue \\ Cambridge, MA 02138 \\ January 2012
}

We are grateful to Michael Bauer, Bryan Brown, Frank Diebold, Ron Gallant, Ken Singleton, anonymous referees, and seminar participants at the University of Chicago, UCSD, Federal Reserve Board, Pennsylvania State University, Society for Financial Econometrics, Midwest Macroeconomics Conference, Rice University, University of Colorado, and the Federal Reserve Bank of San Francisco for comments on earlier drafts of this paper. The views expressed herein are those of the authors and do not necessarily reflect the views of the National Bureau of Economic Research.

NBER working papers are circulated for discussion and comment purposes. They have not been peerreviewed or been subject to the review by the NBER Board of Directors that accompanies official NBER publications.

(C) 2012 by James D. Hamilton and Jing Cynthia Wu. All rights reserved. Short sections of text, not to exceed two paragraphs, may be quoted without explicit permission provided that full credit, including (c) notice, is given to the source. 
Identification and Estimation of Gaussian Affine Term Structure Models

James D. Hamilton and Jing Cynthia Wu

NBER Working Paper No. 17772

January 2012

JEL No. C13,E43,G12

\begin{abstract}
$\underline{\text { ABSTRACT }}$
This paper develops new results for identification and estimation of Gaussian affine term structure models. We establish that three popular canonical representations are unidentified, and demonstrate how unidentified regions can complicate numerical optimization. A separate contribution of the paper is the proposal of minimum-chi-square estimation as an alternative to MLE. We show that, although it is asymptotically equivalent to MLE, it can be much easier to compute. In some cases, MCSE allows researchers to recognize with certainty whether a given estimate represents a global maximum of the likelihood function and makes feasible the computation of small-sample standard errors.
\end{abstract}

James D. Hamilton

Department of Economics, 0508

University of California, San Diego

9500 Gilman Drive

La Jolla, CA 92093-0508

and NBER

jhamilton@ucsd.edu

Jing Cynthia Wu

Booth School of Business

University of Chicago

5807 S Woodlawn Ave

Chicago, IL 60637-1610

Cynthia.Wu@chicagobooth.edu 


\section{Introduction.}

The class of Gaussian affine term structure models ${ }^{1}$ developed by Vasicek (1977), Duffie and Kan (1996), Dai and Singleton (2002), and Duffee (2002) has become the basic workhorse in macroeconomics and finance for purposes of using a no-arbitrage framework for studying the relations between yields on assets of different maturities. Its appeal comes from its simple characterization of how risk gets priced by the market which, under the assumption of no arbitrage, generates predictions for the price of any asset. The approach has been used to measure the role of risk premia in interest rates (Duffee, 2002; Cochrane and Piazzesi, 2009), study how macroeconomic developments and monetary policy affect the term structure of interest rates (Ang and Piazzesi, 2003; Beechey and Wright, 2009; Bauer, 2011), characterize the monetary policy rule (Ang, Dong, and Piazzesi, 2007; Rudebusch and Wu, 2008; Bekaert, Cho, and Moreno, 2010), determine why long-term yields remained remarkably low in 2004 and 2005 (Kim and Wright, 2005; Rudebusch, Swanson, and Wu, 2006), infer market expectations of inflation from the spread between nominal and inflation-indexed Treasury yields (Christensen, Lopez, and Rudebusch, 2010), evaluate the effectiveness of the extraordinary central bank interventions during the financial crisis (Christensen, Lopez, and Rudebusch, 2009; Smith, 2010), and study the potential for monetary policy to affect interest rates when the short rate is at the zero lower bound Hamilton and $\mathrm{Wu}$ (forthcominga).

But buried in the footnotes of this literature and in the practical experience of those who have used these models are tremendous numerical challenges in estimating the necessary

\footnotetext{
${ }^{1}$ By Gaussian affine term structure models we refer to specifications in which the discrete-time joint distribution of yields and factors is multivariate Normal with constant conditional variances. We do not in this paper consider the broader class of non-Gaussian processes.
} 
parameters from the data due to highly non-linear and badly behaved likelihood surfaces. For example, Kim (2008) observed:

Flexibly specified no-arbitrage models tend to entail much estimation difficulty due to a large number of parameters to be estimated and due to the nonlinear relationship between the parameters and yields that necessitates a nonlinear optimization.

Ang and Piazzesi (2003) similarly reported:

difficulties associated with estimating a model with many factors using maximum likelihood when yields are highly persistent....We need to find good starting values to achieve convergence in this highly non-linear system.... [T] he likelihood surface is very flat in $\lambda_{0}$ which determines the mean of long yields....

This paper proposes a solution to these and other problems with affine term structure models based on what we will refer to as their reduced-form representation. For a popular class of Gaussian affine term structure models- namely, those for which the model is claimed to price exactly a subset of $N_{\ell}$ linear combinations of observed yields, where $N_{\ell}$ is the number of unobserved pricing factors- this reduced form is a restricted vector autoregression in the observed set of yields and macroeconomic variables. ${ }^{2}$ We explore two implications of this fact that seem to have been ignored in the large preceding literature on such models.

The first is that the parameters of these reduced-form representations contain all the observable implications of any Gaussian affine term structure model for the sample of observed

\footnotetext{
${ }^{2}$ For more general models where all yields are priced with measurement error, the reduced form is a restricted state-space representation for the set of observed variables. The same tools developed here could still be applied in that setting, though we leave exploration of such models for future research.
} 
data, and can therefore be used as a basis for assessing identification. If more than one value for the parameter vector of interest is associated with the same reduced-form parameter vector, then the model is unidentified at that point and there is no way to use the observed data to distinguish between the alternative possibilities. Although as a general econometric principle this idea dates back to Fisher (1966) and Rothenberg (1971), it has not previously been applied to affine term structure models. In this paper, we use it to demonstrate that the preferred representations proposed by Ang and Piazzesi (2003) and Pericoli and Taboga (2008) are in fact unidentified, an observation that our paper is the first to point out. We also use this approach to show that the representation proposed by Dai and Singleton (2000) is unidentified. Although this latter fact has previously been inferred by Collin-Dufresne, Goldstein, and Jones (2008) and Aït-Sahalia and Kimmel (2010) using other methods, we regard the proof here based on the reduced form to be more transparent and direct. We further demonstrate that it is common for numerical search methods to end up in regions of the parameter space that are locally unidentified, and show why this failure of identification arises. These issues of identification are one factor that contributes to the numerical difficulties for conventional methods noted above.

A second and completely separate contribution of the paper is the observation that it is possible for the parameters of interest to be inferred directly from estimates of the reducedform parameters themselves. This is a very useful result because the latter are often simple OLS coefficients. Although translating from reduced-form parameters into structural parameters involves a mix of analytical and numerical calculations, the numerical component is far simpler than that associated with the usual approach of trying to find the maximum of the 
likelihood surface directly as a function of the structural parameters. In the case of a justidentified structure, the numerical component of our proposed method has an additional big advantage over the traditional approach, in that the researcher knows with certainty whether the equations have been solved, and therefore knows with certainty whether one has found the global maximum of the likelihood surface with respect to the structural parameters or simply a local maximum. In the conventional approach, one instead has to search over hundreds of different starting values, and even then has no guarantee that the global maximum has been found. In the case where the model imposes overidentifying restrictions on the reduced form, one can still estimate structural parameters as functions of the unrestricted reducedform estimates by the method of minimum-chi-square estimation (MCSE). This minimizes a quadratic form in the difference between the reduced-form parameters implied by a given structural model and the reduced-form parameters as estimated without restrictions directly from the data, with the weighting matrix given by the information matrix, in other words, minimizing the value of the chi-square statistic for testing whether the restrictions are indeed consistent with the observed reduced-form estimates.

Again while the general econometric method of minimum-chi-square estimation is well known, our paper is the first to apply it to affine term structure models and demonstrate its considerable advantages in this setting. Estimating parameters by minimizing the chi-square statistic was to our knowledge first proposed by Fisher (1924) and Neyman and Pearson (1928). Rothenberg (1973, pp. 24-25) extended the approach to more general parametric inference, demonstrating that when (as in our proposed application) the reduced-form estimate is the unrestricted MLE and the weighting matrix is the associated information matrix, the resulting 
MCSE is asymptotically equivalent to full-information MLE. MCSE has also been used in other settings by Chamberlain (1982) and Newey (1987).

More generally, MCSE could be viewed as a special case of minimum distance estimation (MDE) discussed for example by Malinvaud (1970), in which one minimizes a quadratic form in the difference between restricted and unrestricted statistics. We follow Rothenberg (1973) in using the expression MCSE to refer to the special case of MDE in which the unrestricted statistics are the unrestricted MLE and weights come from their asymptotic variance, in which case MDE is asymptotically efficient. Another well-known example of MDE is the generalized method of moments (GMM, Hansen (1982)), in which the unrestricted statistics are sample moments. ${ }^{3}$ Bekaert, Cho, and Moreno (2010) used GMM to estimate parameters of an affine term structure model. GMM in this form misses what we see as the two main advantages of MCSE, namely, the OLS estimates are known analytically and MCSE, unlike GMM, is asymptotically efficient.

Another popular example of MDE is the method of indirect inference proposed by Gallant and Tauchen (1992), Smith (1993) and Gourieroux, Monfort, and Renault (1993). With indirect inference, the unrestricted parameter estimates are typically regarded as only approximate or auxiliary characterizations of the data, and numerical simulation is typically required to calculate the values for these auxiliary parameters that are implied by the structural model. Duffee and Stanton (2008) suggested that for highly persistent data such as interest rates, indirect inference or MLE may work substantially better than other moment-based estima-

\footnotetext{
${ }^{3}$ In our application of MCSE, the unrestricted estimates (OLS coefficients and variances) are nonlinear functions of sample moments. This connection between MCSE and GMM is explored further in Chamberlain (1982, p. 18).
} 
tors. One could view our application of MCSE as a special case of indirect inference in which the unrestricted estimates are in fact sufficient statistics for the likelihood function and the mapping from structural parameters to these coefficients is known analytically, precisely the features from which our claimed benefits of MCSE derive.

In particular, we demonstrate in this paper that use of MCSE captures all the asymptotic benefits of MLE while avoiding many of the numerical problems associated with MLE for affine term structure models. Among other illustrations of the computational advantages, we establish the feasibility of calculating small-sample standard errors and confidence intervals for this class of models and demonstrate that the parameter estimates reported by Ang and Piazzesi (2003) in fact correspond to a local maximum of the likelihood surface and are not the global MLE.

There have been several other recent efforts to address many of these problems in affine term structure models. Christensen, Diebold, and Rudebusch (2011) developed a no-arbitrage representation of a dynamic Nelson-Siegel model of interest rates that gives a convenient representation of level, slope and curvature factors and offers significant improvements in empirical tractability and predictive performance over earlier affine term structure specifications. Joslin, Singleton, and Zhu (2011) proposed a canonical representation for affine term structure models that greatly improves convergence of maximum likelihood estimation. Collin-Dufresne, Goldstein, and Jones (2008) proposed a representation in terms of the derivatives of the term structure at maturity zero, arguing for the benefits of using these observable magnitudes rather than unobserved latent variables to represent the state vector of an ATSM. Each of these papers proposes canonical representations that are identified, and the Christensen, Diebold, 
and Rudebusch (2011) and Joslin, Singleton, and Zhu (2011) parameterizations lead to better behaved likelihood functions than do the parameterizations explored in detail in our paper.

The chief difference between our proposed solution and those of these other researchers is that they focus on how the ATSM should be represented, whereas we examine how the parameters of the ATSM are to be estimated. Thus for example Christensen, Diebold, and Rudebusch (2011) require the researcher to impose certain restrictions on the ATSM, whereas Joslin, Singleton, and Zhu (2011) cannot incorporate most auxiliary restrictions on the $P$ dynamics. It is far from clear how any of these three approaches could have been used to estimate a model of the form investigated by Ang and Piazzesi (2003). By contrast, our MCSE algorithm can be used for any representation, including those proposed by Christensen, Diebold, and Rudebusch (2011) and Joslin, Singleton, and Zhu (2011), and can simplify the numerical burden regardless of the representation chosen. Indeed, some of the numerical advantages of Joslin, Singleton, and Zhu (2011) come from the fact that a subset of their parameterization is identical to a subset of our reduced-form representation, and their approach, like ours, takes advantage of the fact that the full-information MLE for this subset can be obtained by OLS for a popular class of models. However, Joslin, Singleton, and Zhu (2011) estimated the remaining parameters by conventional MLE rather than using the full set of reduced-form estimates as in our approach. As Joslin, Singleton, and Zhu (2011) noted, their representation becomes unidentified in the presence of a unit root. When applied to highly persistent data, we illustrate that their MLE algorithm can encounter similar problems to those of other representations, which can be avoided with our approach to parameter estimation.

The rest of the paper is organized as follows. Section 2 describes the class of Gaussian affine 
term structure models and three popular examples, and briefly uses one of the specifications to illustrate the numerical difficulties that can be encountered with the traditional approach. Section 3 investigates the mapping from structural to reduced-form parameters. We establish that the canonical forms of all three examples are unidentified and explore how this contributes to some of the problems for conventional numerical search algorithms. In Section 4 we use the mapping to propose approaches to parameter estimation that are much better behaved. Section 5 concludes.

\section{Gaussian Affine Term Structure Models.}

\subsection{Basic framework}

Consider an $(M \times 1)$ vector of variables $F_{t}$ whose dynamics are characterized by a Gaussian vector autoregression:

$$
F_{t+1}=c+\rho F_{t}+\Sigma u_{t+1}
$$

with $u_{t} \sim$ i.i.d. $N\left(0, I_{M}\right)$. This specification implies that $F_{t+1} \mid F_{t}, F_{t-1}, \ldots, F_{1} \sim N\left(\mu_{t}, \Sigma \Sigma^{\prime}\right)$ for

$$
\mu_{t}=c+\rho F_{t} .
$$

Let $r_{t}$ denote the risk-free one-period interest rate. If the vector $F_{t}$ includes all the variables that could matter to investors, then the price of a pure discount asset at date $t$ should be a function $P_{t}\left(F_{t}\right)$ of the current state vector. Moreover, if investors were risk neutral, the price 
they'd be willing to pay would satisfy

$$
\begin{aligned}
P_{t}\left(F_{t}\right) & =\exp \left(-r_{t}\right) E_{t}\left[P_{t+1}\left(F_{t+1}\right)\right] \\
& =\exp \left(-r_{t}\right) \int_{\mathbb{R}^{M}} P_{t+1}\left(F_{t+1}\right) \phi\left(F_{t+1} ; \mu_{t}, \Sigma \Sigma^{\prime}\right) d F_{t+1}
\end{aligned}
$$

for $\phi(y ; \mu, \Omega)$ the $M$-dimensional $N(\mu, \Omega)$ density evaluated at the point $y$ :

$$
\phi(y ; \mu, \Omega)=\frac{1}{(2 \pi)^{M / 2}|\Omega|^{1 / 2}} \exp \left[-\frac{(y-\mu)^{\prime} \Omega^{-1}(y-\mu)}{2}\right] .
$$

More generally, with risk-averse investors we would replace (3) with

$$
\begin{aligned}
P_{t}\left(F_{t}\right) & =E_{t}\left[P_{t+1}\left(F_{t+1}\right) M_{t, t+1}\right] \\
& =\int_{\mathbb{R}^{M}} P_{t+1}\left(F_{t+1}\right)\left[M_{t, t+1} \phi\left(F_{t+1} ; \mu_{t}, \Sigma \Sigma^{\prime}\right)\right] d F_{t+1}
\end{aligned}
$$

for $M_{t, t+1}$ the pricing kernel. In many macro models, the pricing kernel would be

$$
M_{t, t+1}=\frac{\beta U^{\prime}\left(C_{t+1}\right)}{U^{\prime}\left(C_{t}\right)\left(1+\pi_{t+1}\right)}
$$

for $\beta$ the personal discount rate, $U^{\prime}(C)$ the marginal utility of consumption, and $\pi_{t+1}$ the inflation rate between $t$ and $t+1$.

Affine term structure models are derived from the particular kernel

$$
M_{t, t+1}=\exp \left[-r_{t}-(1 / 2) \lambda_{t}^{\prime} \lambda_{t}-\lambda_{t}^{\prime} u_{t+1}\right]
$$


for $\lambda_{t}$ an $(M \times 1)$ vector that characterizes investor attitudes toward risk, with $\lambda_{t}=0$ in the case of risk neutrality. Elementary multiplication of (4) by (6) reveals that for this case

$$
M_{t, t+1} \phi\left(F_{t+1} ; \mu_{t}, \Sigma \Sigma^{\prime}\right)=\exp \left(-r_{t}\right) \phi\left(F_{t+1} ; \mu_{t}^{Q}, \Sigma \Sigma^{\prime}\right)
$$

for

$$
\mu_{t}^{Q}=\mu_{t}-\Sigma \lambda_{t}
$$

Substituting (7) into (5) and comparing with (3), we see that for this specification of the pricing kernel, risk-averse investors value any asset the same as risk-neutral investors would if the latter thought that the conditional mean of $F_{t+1}$ was $\mu_{t}^{Q}$ rather than $\mu_{t}$. A positive value for the first element of $\lambda_{t}$, for example, implies that an asset that delivers the quantity $F_{1, t+1}$ dollars in period $t+1$ would have a value at time $t$ that is less than the value that would be assigned by a risk-neutral investor, and the size of this difference is bigger when the $(1,1)$ element of $\Sigma$ is bigger. An asset yielding $F_{i, t+1}$ dollars has a market value that is reduced by $\Sigma_{i 1} \lambda_{1 t}$ relative to a risk-neutral valuation, through the covariance between factors $i$ and 1 . The term $\lambda_{1 t}$ might then be described as the market price of factor 1 risk.

The affine term structure models further postulate that this market price of risk is itself an affine function of $F_{t}$,

$$
\lambda_{t}=\lambda+\Lambda F_{t}
$$

for $\lambda$ an $(M \times 1)$ vector and $\Lambda$ an $(M \times M)$ matrix. Substituting (9) and (2) into (8), we see 
that

$$
\mu_{t}^{Q}=c^{Q}+\rho^{Q} F_{t}
$$

for

$$
\begin{gathered}
c^{Q}=c-\Sigma \lambda \\
\rho^{Q}=\rho-\Sigma \Lambda .
\end{gathered}
$$

In other words, risk-averse investors value assets the same way as a risk-neutral investor would if that risk-neutral investor believed that the factors are characterized by a $Q$-measure VAR given by

$$
F_{t+1}=c^{Q}+\rho^{Q} F_{t}+\Sigma u_{t+1}^{Q}
$$

with $u_{t+1}^{Q}$ a vector of independent standard Normal variables under the $Q$ measure.

Suppose that the risk-free 1-period yield is also an affine function of the factors

$$
r_{t}=\delta_{0}+\delta_{1}^{\prime} F_{t}
$$

Then, as demonstrated for example in Appendix A of Ang and Piazzesi (2003), under the above assumptions the yield on a risk-free $n$-period pure-discount bond can be calculated as

$$
y_{t}^{n}=a_{n}+b_{n}^{\prime} F_{t}
$$


where

$$
\begin{aligned}
b_{n}= & \frac{1}{n}\left[I_{M}+\rho^{Q \prime}+\cdots+\left(\rho^{Q \prime}\right)^{n-1}\right] \delta_{1} \\
a_{n}= & \delta_{0}+\left(b_{1}^{\prime}+2 b_{2}^{\prime}+\cdots+(n-1) b_{n-1}^{\prime}\right) c^{Q} / n \\
& -\left(b_{1}^{\prime} \Sigma \Sigma^{\prime} b_{1}+2^{2} b_{2}^{\prime} \Sigma \Sigma^{\prime} b_{2}+\cdots+(n-1)^{2} b_{n-1}^{\prime} \Sigma \Sigma^{\prime} b_{n-1}\right) / 2 n .
\end{aligned}
$$

If we knew $F_{t}$ and the values of $c^{Q}$ and $\rho^{Q}$ along with $\delta_{0}, \delta_{1}$, and $\Sigma$, we could use (14), (15), and (16) to predict the yield for any maturity $n$.

There are thus three sets of parameters that go into an affine term structure model: (a) the parameters $c, \rho$, and $\Sigma$ that characterize the objective dynamics of the factors in equation (1) (sometimes called the $P$ parameters); (b) the parameters $\lambda$ and $\Lambda$ in equation (9) that characterize the price of risk; and (c) the $Q$ parameters $c^{Q}$ and $\rho^{Q}$ (along with the same $\Sigma$ as appeared in the $P$ parameter set) that figure in (12). If we knew any two of these sets of parameters, we could calculate the third ${ }^{4}$ using (10) and (11). We will refer to a representation in terms of (a) and (b) as a $\lambda$ representation, and a representation in terms of (a) and (c) as a $Q$ representation.

Suppose we want to describe yields on a set of $N_{d}$ different maturities. If $N_{d}$ is greater than $N_{\ell}$, where $N_{\ell}$ is the number of unobserved pricing factors, then (14) would imply that it should be possible to predict the value of one of the $y_{n t}$ as an exact linear function of the others. Although in practice we can predict one yield extremely accurately given the others, the empirical fit is never exact. One common approach to estimation, employed for

\footnotetext{
${ }^{4}$ We will discuss examples below in which $\Sigma$ is singular for which the demonstration of this equivalence is a bit more involved, with the truth of the assertion coming from the fact that for such cases certain elements of $\lambda$ and $\Lambda$ are defined to be zero.
} 
example by Ang and Piazzesi (2003) and Chen and Scott (1993), is to suppose that (14) holds exactly for $N_{\ell}$ linear combinations of observed yields, and that the remaining $N_{e}=N_{d}-N_{\ell}$ linear combinations differ from the predicted value by a small measurement error. Let $Y_{t}^{1}$ denote the $\left(N_{\ell} \times 1\right)$ vector consisting of those linear combinations of yields that are treated as priced without error and $Y_{t}^{2}$ the remaining $\left(N_{e} \times 1\right)$ linear combinations. The measurement specification is then

$$
\left[\begin{array}{c}
Y_{t}^{1} \\
\left(N_{\ell} \times 1\right) \\
Y_{t}^{2} \\
\left(N_{e} \times 1\right)
\end{array}\right]=\left[\begin{array}{c}
A_{1} \\
\left(N_{\ell} \times 1\right) \\
A_{2} \\
\left(N_{e} \times 1\right)
\end{array}\right]+\left[\begin{array}{c}
B_{1} \\
\left(N_{\ell} \times M\right) \\
B_{2} \\
\left(N_{e} \times M\right)
\end{array}\right] F_{t}+\left[\begin{array}{c}
0 \\
\left(N_{\ell} \times N_{e}\right) \\
\Sigma_{e} \\
\left(N_{e} \times N_{e}\right)
\end{array}\right] \begin{gathered}
u_{t}^{e} \\
\left(N_{e} \times 1\right)
\end{gathered}
$$

where $\Sigma_{e}$ is typically taken to be diagonal. Here $A_{i}$ and $B_{i}$ are calculated by stacking (16) and (15), respectively, for the appropriate $n$, while $\Sigma_{e}$ determines the variance of the measurement error with $u_{t}^{e} \sim N\left(0, I_{N_{e}}\right)$. We will discuss many of the issues associated with identification and estimation of affine term structure models in terms of three examples.

\subsection{Example 1: Latent factor model.}

In this specification, the factors $F_{t}$ governing yields are treated as if observable only through their implications for the yields themselves; examples in the continuous-time literature include Dai and Singleton (2000), Duffee (2002), and Kim and Orphanides (2005). Typically in this case, the number of factors $N_{\ell}$ and the number of yields observed without error are both taken to be 3 , with the 3 factors interpreted as the level, slope, and curvature of the term structure. The 3 linear combinations $Y_{t}^{1}$ regarded as observed without error can be constructed from 
the first 3 principal components of the set of yields. Alternatively, they could be constructed directly from logical measures of level, slope, and curvature. Yet another option is simply to choose 3 representative yields as the elements of $Y_{t}^{1}$. Which linear combinations are claimed to be priced without error can make a difference for certain testable implications of the model, an issue that we explore in a separate paper (Hamilton and $\mathrm{Wu}$, forthcomingb) which addresses empirical testing of the overidentifying restrictions of affine term structure models. For purposes of discussing identification and estimation, however, the choice of which yields go into $Y_{t}^{1}$ is immaterial, and notation is kept simplest by following Ang and Piazzesi (2003) and Pericoli and Taboga (2008) in just using 3 representative yields. In our numerical example, these are taken to be the $n=1-, 12-$, and 60 -month maturities, with data on 36month yields included separately in $Y_{t}^{2}$. Thus for this illustrative latent-factor specification, equation (17) takes the form

$$
\left[\begin{array}{c}
y_{t}^{1} \\
y_{t}^{12} \\
y_{t}^{60} \\
y_{t}^{36}
\end{array}\right]=\left[\begin{array}{c}
a_{1} \\
a_{12} \\
a_{60} \\
a_{36}
\end{array}\right]+\left[\begin{array}{c}
b_{1}^{\prime} \\
b_{12}^{\prime} \\
b_{60}^{\prime} \\
b_{36}^{\prime}
\end{array}\right] F_{t}+\left[\begin{array}{c}
0 \\
0 \\
0 \\
\Sigma_{e}
\end{array}\right] u_{t}^{e}
$$

where $a_{n}$ and $b_{n}$ are calculated from equations (16) and (15), respectively.

We will use for our illustration a $Q$ representation for this system. Dai and Singleton (2000) proposed the normalization conditions $\Sigma=I_{N_{\ell}}, \delta_{1} \geq 0, c=0$ and $\rho$ lower triangular. Singleton (2006) used parallel constraints on the $Q$ parameters $\left(\Sigma=I_{N_{\ell}}, \delta_{1} \geq 0, c^{Q}=0, \rho^{Q}\right.$ lower triangular). Our illustration will use $\Sigma=I_{N_{\ell}}, \delta_{1} \geq 0, c=0$ and $\rho^{Q}$ lower triangular. 
For the $N_{\ell}=3, N_{e}=1$ case displayed in equation (18), there are then 23 unknown parameters: 3 in $c^{Q}, 6$ in $\rho^{Q}, 9$ in $\rho, 1$ in $\delta_{0}, 3$ in $\delta_{1}$, and 1 in $\Sigma_{e}$, which we collect in the $(23 \times 1)$ vector $\theta$. The log likelihood is

$$
\mathcal{L}(\theta ; Y)=\sum_{t=1}^{T}\left\{-\log [|\operatorname{det}(J)|]+\log \phi\left(F_{t} ; c+\rho F_{t-1}, I_{N_{\ell}}\right)+\log \phi\left(u_{t}^{e} ; 0, I_{N_{e}}\right)\right\}
$$

for $\phi($.$) the multivariate Normal density in equation (4) and \operatorname{det}(J)$ the determinant of the Jacobian, with

$$
\begin{gathered}
J=\left[\begin{array}{cc}
B_{1} & 0 \\
\left(N_{\ell} \times N_{\ell}\right) & \left(N_{\ell} \times N_{e}\right) \\
B_{2} & \Sigma_{e} \\
\left(N_{e} \times N_{\ell}\right) & \left(N_{e} \times N_{e}\right)
\end{array}\right] \\
F_{t}=B_{1}^{-1}\left(Y_{t}^{1}-A_{1}\right) \\
u_{t}^{e}=\Sigma_{e}^{-1}\left\{Y_{t}^{2}-A_{2}-B_{2} B_{1}^{-1}\left(Y_{t}^{1}-A_{1}\right)\right\} .
\end{gathered}
$$

The Chen-Scott procedure is to maximize (19) with respect to $\theta$ by numerical search.

As a simple example to illustrate the difficulties with this traditional estimation and some of the advantages of the procedure that we will be recommending to replace it, we simulated a sample of 1000 observations using parameters specified in the first block of Table 1 below. These parameters were chosen to match the actual observed behavior of the four yields used here. On this sample we tried to choose $\theta$ so as to maximize (19) using the fminunc algorithm in MATLAB. ${ }^{5}$ Since numerical search can be sensitive to different scaling of parameters, we

\footnotetext{
${ }^{5}$ MATLAB numerical optimizers have been used by Cochrane and Piazzesi (2009), Aït-Sahalia and Kimmel (2010), and Joslin, Singleton, and Zhu (2011), among others. Duffee (2011) found that numerical search problems can be reduced using alternative algorithms. Our purpose here is to illustrate the difficulties that can arise in estimation. We will demonstrate that these identical MATLAB algorithms have no trouble with the alternative formulation that we will propose below.
} 
tried to scale parameters in a way consistent with a researcher's prior expectation that risk prices were small, multiplying $c^{Q}$ by 10 and $\delta_{1}$ and $\Sigma_{e}$ by 1000 so that a unit step for each of these parameters would be similar to a unit step for the others. ${ }^{6}$ We used 100 different starting values for this search, using a range of values for $\rho^{Q}$ and starting the other parameters at good guesses. Specifically, to obtain a given starting value we would generate the 3 diagonal elements of $\rho^{Q}$ from $U[0.5,1]$ distributions, set off-diagonal elements to zero, and set the initial guess for $\rho$ equal to this value for $\rho^{Q}$. We set the starting value for each element of $\delta_{1}$ and $\Sigma_{e}$ to $1 . \mathrm{e}-4, \delta_{0}=0.0046$ (the average short rate), and $c^{Q}=0$.

In only 1 of these 100 experiments did the numerical search converge to the values that we will establish below are indeed the true global MLE. These estimates, reported in the second block of Table 1, in fact correspond very nicely to the true values from which this sample was simulated. However, in 81 of the other experiments, the procedure satisfied the convergence criterion (usually coming from a sufficiently tiny change between iterations) at a large range of alternative points other than the global maximum. The third block of Table 1 displays one of these. All such points are characterized by an eigenvalue of $\rho$ being equal or very close to unity; we will explain why this happens in the following section. For the other 18 starting values, the search algorithm was unable to make any progress from the initial starting values. Although very simple, this exercise helps convey some sense of the numerical problems researchers have encountered fitting more complicated models such as we describe in our next two examples.

\footnotetext{
${ }^{6}$ To give the algorithm the best chance to converge, for each starting value we allowed the search to continue for up to 10,000 function evaluations, then restarted the search at that terminal value to allow an additional 10,000 function evaluations, and so on, for 10 repetitions with each starting value.
} 


\subsection{Example 2: Macro finance model with single lag (MF1).}

It is of considerable interest to include observable macroeconomic variables among the factors that may affect interest rates, as for example in Ang and Piazzesi (2003), Ang, Dong, and Piazzesi (2007), Rudebusch and Wu (2008), Ang, Piazzesi, and Wei (2006), and Hördahl, Tristani, and Vestin (2006). Our next two illustrative examples come from this class. We first consider the unrestricted first-order macro factor model studied by Pericoli and Taboga (2008). This model uses $N_{m}=2$ observable macro factors, consisting of measures of the inflation rate and the output gap, which are collected in an $\left(N_{m} \times 1\right)$ vector $f_{t}^{m}$. These two observable macroeconomic factors are allowed to influence yield dynamics in addition to the traditional $N_{\ell}=3$ latent $^{7}$ factors $f_{t}^{\ell}$,

$$
\underset{\left(N_{f} \times 1\right)}{F_{t}}=\left[\begin{array}{c}
f_{t}^{m} \\
\left(N_{m} \times 1\right) \\
f_{t}^{\ell} \\
\left(N_{\ell} \times 1\right)
\end{array}\right],
$$

for $N_{f}=N_{m}+N_{\ell}$. The $P$ dynamics (1), $Q$ dynamics (12), and short-rate equation (13) can for this example be written in partitioned form as

$$
\begin{aligned}
\underset{\left(N_{m} \times 1\right)}{f_{t}^{m}} & =c_{m}+\rho_{m m} f_{t-1}^{m}+\rho_{m \ell} f_{t-1}^{\ell}+\Sigma_{m m} u_{t}^{m} \\
\underset{\left(N_{\ell} \times 1\right)}{f_{t}^{\ell}} & =c_{\ell}+\rho_{\ell m} f_{t-1}^{m}+\rho_{\ell \ell} f_{t-1}^{\ell}+\Sigma_{\ell m} u_{t}^{m}+\Sigma_{\ell \ell} u_{t}^{\ell}
\end{aligned}
$$

\footnotetext{
${ }^{7}$ Pericoli and Taboga evaluated a number of alternative specifications including different choices for the number of latent factors $N_{\ell}$, number of lags on the macro variables, and dependence between the latent and macro factors. They refer to the specification we discuss in the text as the $M(3,0, U)$ specification, which is the one that their tests suggest best fits the data.
} 


$$
\begin{aligned}
& \underset{\left(N_{m} \times 1\right)}{f_{t}^{m}}=c_{m}^{Q}+\rho_{m m}^{Q} f_{t-1}^{m}+\rho_{m \ell}^{Q} f_{t-1}^{\ell}+\Sigma_{m m} u_{t}^{Q m} \\
& \underset{\left(N_{\ell} \times 1\right)}{f_{t}^{\ell}}=c_{\ell}^{Q}+\rho_{\ell m}^{Q} f_{t-1}^{m}+\rho_{\ell \ell}^{Q} f_{t-1}^{\ell}+\Sigma_{\ell m} u_{t}^{Q m}+\Sigma_{\ell \ell} u_{t}^{Q \ell} \\
& r_{t}=\delta_{0}+\delta_{1 m}^{\prime} f_{t}^{m}+\delta_{1 \ell}^{\prime} f_{t}^{\ell}
\end{aligned}
$$

Pericoli and Taboga proposed the normalization conditions ${ }^{8}$ that $\Sigma_{m m}$ is lower triangular, $\Sigma_{\ell m}=0, \Sigma_{\ell \ell}=I_{N_{\ell}}, \delta_{1 \ell} \geq 0$, and $c_{\ell}^{Q}=0$.

Our empirical illustration of this approach will use $t$ corresponding to quarterly data and will take the 1-, 5-, and 10-year bonds to be priced without error $\left(Y_{t}^{1}=\left(y_{t}^{4}, y_{t}^{20}, y_{t}^{40}\right)^{\prime}\right)$ and the 2-, 3-, and 7-year bonds to be priced with error $\left(Y_{t}^{2}=\left(y_{t}^{8}, y_{t}^{12}, y_{t}^{28}\right)^{\prime}\right)$. Details of how the log likelihood is calculated for this example are described in Appendix A.

\subsection{Example 3: Macro finance model with 12 lags (MF12).}

A first-order VAR is not sufficient to capture the observed dynamics of output and inflation.

For example, Ang and Piazzesi (2003) suggested that the best fit is obtained using a monthly $\operatorname{VAR}(12)$ in the observable macro variables and a $\operatorname{VAR}(1)$ for the latent factors: ${ }^{9}$

$$
\begin{aligned}
f_{t}^{m} & =\rho_{1} f_{t-1}^{m}+\rho_{2} f_{t-2}^{m}+\cdots+\rho_{12} f_{t-12}^{m}+\Sigma_{m m} u_{t}^{m} \\
f_{t}^{\ell} & =c_{\ell}+\rho_{\ell \ell} f_{t-1}^{\ell}+\Sigma_{\ell \ell} u_{t}^{\ell} .
\end{aligned}
$$

\footnotetext{
${ }^{8}$ Pericoli and Taboga imposed $f_{0}^{\ell}=0$ as an alternative to the traditional $c_{\ell}=0$ or $c_{\ell}^{Q}=0$, though we will follow the rest of the literature here in using a more standard normalization.

${ }^{9}$ Ang and Piazzesi refer to this as their Macro Model.
} 
Our empirical example follows Ang and Piazzesi in proxying the 2 elements of $f_{t}^{m}$ with the first principal components of a set of output and and a set of inflation measures, respectively, which factors have mean zero by construction. Ang and Piazzesi treated the macro dynamics as independent of those for the unobserved latent factors, so that terms such as $\rho_{\ell m}$ and $\rho_{m \ell}$ in the preceding example are set to zero.

Ang and Piazzesi (2003) further proposed the following identifying restrictions: $\Sigma_{m m}$ is lower triangular, $\Sigma_{\ell \ell}=I_{N_{\ell}}, c_{\ell}=0, \rho_{\ell \ell}$ is lower triangular, and the diagonal elements of $\rho_{\ell \ell}$ are in descending order. Further restrictions and details of the model and its likelihood function are provided in Appendix B. In the specification we replicate, Ang and Piazzesi postulated that the short rate depends only on the current values of the macro factors:

$$
r_{t}=\delta_{0}+\delta_{1 m}^{\prime} f_{t}^{m}+\delta_{1 \ell}^{\prime} f_{t}^{\ell}
$$

They further noted that since $f_{t}^{\ell}$ is independent of $f_{t}^{m}$ under their assumptions, the values of $\delta_{0}$ and $\delta_{1 m}$ in the short-rate equation can be obtained by OLS estimation of

$$
r_{t}=\delta_{0}+\delta_{1 m}^{\prime} f_{t}^{m}+v_{t}
$$

To further reduce the dimensionality of the estimation, Ang and Piazzesi (2003) proposed some further restrictions on this set-up that we will discuss in more detail in Section 4.4. 


\section{Identification.}

The log likelihood function for each of the models discussed- and indeed, for any Gaussian affine term structure model in which exactly $N_{\ell}$ linear combinations of yields are assumed to be priced without error- takes the form of a restricted vector autoregression. The mapping from the affine-pricing parameters to the VAR parameters allows us to evaluate the identifiability

of a given structure. If two different values for the structural parameters imply the identical reduced-form parameters, there is no way to use observable data to choose between the two. We now explore the implications of this fact for each of the three classes of models described in the previous section.

\subsection{Example 1: Latent factor model.}

Premultiplying (1) by $B_{1}$ (and recalling the normalization $c=0$ and $\Sigma=I_{N_{\ell}}$ ) results in

$$
B_{1} F_{t}=B_{1} \rho B_{1}^{-1} B_{1} F_{t-1}+B_{1} u_{t}
$$

Adding $A_{1}$ to both sides and substituting $Y_{t}^{1}=A_{1}+B_{1} F_{t}$ establishes

$$
\begin{gathered}
Y_{t}^{1}=A_{1}^{*}+\phi_{11}^{*} Y_{t-1}^{1}+u_{1 t}^{*} \\
A_{1}^{*}=A_{1}-B_{1} \rho B_{1}^{-1} A_{1} \\
\phi_{11}^{*}=B_{1} \rho B_{1}^{-1} .
\end{gathered}
$$


Likewise the second block of (17) implies

$$
\begin{gathered}
Y_{t}^{2}=A_{2}^{*}+\phi_{21}^{*} Y_{t}^{1}+u_{2 t}^{*} \\
A_{2}^{*}=A_{2}-B_{2} B_{1}^{-1} A_{1} \\
\phi_{21}^{*}=B_{2} B_{1}^{-1} \\
{\left[\begin{array}{c}
u_{1 t}^{*} \\
u_{2 t}^{*}
\end{array}\right] \sim N\left(\left[\begin{array}{l}
0 \\
0
\end{array}\right],\left[\begin{array}{cc}
\Omega_{1}^{*} & 0 \\
0 & \Omega_{2}^{*}
\end{array}\right]\right)} \\
\Omega_{1}^{*}=B_{1} B_{1}^{\prime} \\
\Omega_{2}^{*}=\Sigma_{e} \Sigma_{e}^{\prime} .
\end{gathered}
$$

Equations (24) and (27) will be recognized as a restricted Gaussian VAR for $Y_{t}$, in which a single lag of $Y_{t-1}^{1}$ appears in the equation for $Y_{t}^{1}$ and in which, after conditioning on the contemporaneous value of $Y_{t}^{1}$, no lagged terms appear in the equation for $Y_{t}^{2}$. Note that when we refer to the reduced-form for this system, we will incorporate those exclusion restrictions along with the restriction that $\Omega_{2}^{*}$ is diagonal.

Table 2 summarizes the mapping between the VAR parameters and the affine term structure parameters implied by equations (24)-(32). ${ }^{10} \quad$ The number of VAR parameters minus the number of structural parameters is equal to $\left(N_{e}-1\right)\left(N_{\ell}+1\right)$. Thus the structure is just-identified by a simple parameter count when $N_{e}=1$ and overidentified when $N_{e}>1$. Notwithstanding, the structural parameters can nevertheless be unidentified despite the ap-

\footnotetext{
${ }^{10}$ The value of $\delta_{1}$ turns out not to appear in the product $\phi_{21}^{*}=B_{2} B_{1}^{-1}$.
} 
parent conclusion from a simple parameter count.

Consider first what happens at a point where one of the eigenvalues of $\rho$ is unity, that is, when the $P$-measure factor dynamics exhibit a unit root. ${ }^{11}$ This means that one of the eigenvalues of $B_{1} \rho B_{1}^{-1}$ is also unity $\left(B_{1} \rho B_{1}^{-1} x=x\right.$ for some nonzero $\left.x\right)$ requiring that $\left(I_{N_{\ell}}-B_{1} \rho B_{1}^{-1}\right) x=0$, so the matrix $I_{N_{\ell}}-B_{1} \rho B_{1}^{-1}$ is noninvertible. In this case, even if we knew the true value of $A_{1}^{*}$, we could never find the value of $A_{1}$ from equation (25). If $\hat{A}_{1}$ is proposed as a fit for a given sample, then $\hat{A}_{1}+k x$ produces the identical fit for any $k$. Note moreover from (16) that $A_{1}$ and $A_{2}$ are the only way to find out about $c^{Q}$ and $\delta_{0}$; if we don't know the 4 values in $A_{1}$ and $A_{2}$, we can never infer the 4 values of $c^{Q}$ and $\delta_{0}$. This failure of local identification accounts for the numerous failed searches described in Section 2.2. When the search steps in a region in which $\rho$ has a near unit root, the likelihood surface becomes extremely flat in one direction (and exactly flat at the unit root), causing the numerical search to become bogged down. Because the true process is quite persistent, it is extremely common for a numerical search to explore this region of the surface and become stuck. ${ }^{12}$

If instead we used the normalization $c^{Q}=0$ in place of the condition $c=0$ just analyzed, a similar phenomenon occurs in which a unit root in $\rho^{Q}$ results in a failure of local identification of $\delta_{0}$.

Even when all eigenvalues of $\rho$ are less than unity, there is another respect in which the latent factor model discussed here is unidentified. ${ }^{13}$ Let $H$ denote any $\left(N_{\ell} \times N_{\ell}\right)$ matrix such

\footnotetext{
${ }^{11}$ Note we have followed Ang and Piazzesi (2003) and Joslin, Singleton, and Zhu (2011), among others, in basing estimates on the likelihood function conditional on the first observation. By contrast, Chen and Scott (1993) and Duffee (2002) included the unconditional likelihood of the first observation as a device for imposing stationarity.

${ }^{12}$ This point has also been made by Aït-Sahalia and Kimmel (2010).

${ }^{13}$ This has also been recognized by Ang and Piazzesi (2003), Collin-Dufresne, Goldstein, and Jones (2008) and Aït-Sahalia and Kimmel (2010).
} 
that $H^{\prime} H=I_{N_{\ell}}$. It is apparent from equations (24)-(32) that if we replace $B_{j}$ by $B_{j} H^{\prime}$ and $\rho$ by $H \rho H^{\prime}$, there would be no change in the implied value for the sample likelihood. The question then is whether the conditions imposed on the underlying model rule out such a transformation. From equation (16), such a transformation requires replacing $c^{Q}$ with $H c^{Q}$, and from (15) we need now to use $H \delta_{1}$ and $H \rho^{Q} H^{\prime}$. Since our specification imposed no restrictions on $\rho$ or $c^{Q}$, the question is whether the proposed lower triangular structure for $\rho^{Q}$ and nonnegativity of $\delta_{1}$ rules out such a transformation. The following proposition establishes that it does not.

Proposition 1. Consider any $(2 \times 2)$ lower triangular matrix:

$$
\rho^{Q}=\left[\begin{array}{cc}
\rho_{11}^{Q} & 0 \\
\rho_{21}^{Q} & \rho_{22}^{Q}
\end{array}\right]
$$

Then for almost all $(2 \times 1)$ positive vectors $\delta_{1}$, there exists a unique orthogonal matrix $H$ other than the identity matrix such that $H \rho^{Q} H^{\prime}$ is also lower triangular and $H \delta_{1}>0$. Moreover, $H \rho^{Q} H^{\prime}$ takes one of the following forms:

$$
\left[\begin{array}{cc}
\rho_{22}^{Q} & 0 \\
\rho_{21}^{Q} & \rho_{11}^{Q}
\end{array}\right] \text { or }\left[\begin{array}{cc}
\rho_{22}^{Q} & 0 \\
-\rho_{21}^{Q} & \rho_{11}^{Q}
\end{array}\right]
$$

For $\rho^{Q}$ an $\left(N_{\ell} \times N_{\ell}\right)$ lower triangular matrix, there are $N_{\ell}$ ! different lower triangular representations, characterized by alternative orderings of the principal diagonal elements.

There thus exist 6 different parameter configurations that would achieve the same maximum for the likelihood function for the latent example explored in Section 2.2. The experiment 
did not uncover them because the other difficulties with maximization were sufficiently severe that for the 100 different starting values used, only one of these 6 configurations was reached. Dai and Singleton (2000) and Singleton (2006) originally proposed lower triangularity of $\rho$ or $\rho^{Q}$ and nonnegativity of $\delta_{1}$ as sufficient identifying conditions. Our proposition establishes that one needs a further condition such as $\rho_{11}^{Q} \geq \rho_{22}^{Q} \geq \rho_{33}^{Q}$ to have a globally identified structure.

Nevertheless, this multiplicity of global optima is a far less serious problem than the failure of local identification arising from a unit root. The reason is that any of the alternative configurations obtained through these $H$ transformations by construction has the identical implications for bond pricing. By contrast, the inferences one would draw from Local 53 in Table 1 are fundamentally flawed and introduce substantial practical difficulties for using this class of models.

There is another identification issue, which has separately been recognized by Joslin, Singleton, and Zhu (2011) using a very different approach from ours: not all matrices $\rho^{Q}$ can be transformed into lower triangular form. For example, for $N_{\ell}=2$, if $\rho^{Q}$ is written as lower triangular, then $\rho_{22}^{Q}$ would have to be one of its eigenvalues. However, it is possible for an unrestricted real-valued matrix $\rho^{Q}$ to have complex eigenvalues, in which case there is no way to transform it as $\Upsilon=H \rho^{Q} H^{\prime}$ for $\Upsilon$ a real-valued lower triangular matrix. We propose in the following proposition an alternative normalization for the case $N_{\ell}=2$ that, unlike the usual lower-triangular form, is completely unrestrictive. 
Proposition 2. Consider $\rho^{Q}$ any $(2 \times 2)$ real-valued matrix:

$$
\rho^{Q}=\left[\begin{array}{cc}
\rho_{11}^{Q} & \rho_{12}^{Q} \\
\rho_{21}^{Q} & \rho_{22}^{Q}
\end{array}\right]
$$

For almost all $\delta_{1} \in \mathbb{R}^{2+}$, there exist exactly two transformations of the form $\Upsilon=H \rho^{Q} H^{\prime}$ such that $\Upsilon$ is real, $H^{\prime} H=I_{2}, H \delta_{1}>0$, and the two elements on the principal diagonal of $\Upsilon$ are the same. Moreover, one of these transformations is simply the transpose of the other:

$$
\Upsilon_{1}=\left[\begin{array}{ll}
a & b \\
c & a
\end{array}\right] \quad \Upsilon_{2}=\left[\begin{array}{ll}
a & c \\
b & a
\end{array}\right]
$$

Hence one approach for the $N_{\ell}=2$ case would be to choose the 3 parameters $a$, $b$, and $c$ so as to maximize the likelihood with

$$
\rho^{Q}=\left[\begin{array}{ll}
a & b \\
c & a
\end{array}\right]
$$

subject to the normalization $b \leq c$. This has the advantage over the traditional lowertriangular formulation in that the latter imposes additional restrictions on the dynamics (namely, lower-triangular $\rho^{Q}$ rules out the possibility of complex roots) whereas the $\Upsilon$ formulation does not.

Unfortunately, it is less clear how to generalize this to larger dimensions. If $\rho^{Q}$ has complex eigenvalues, these always appear as complex conjugates. Thus if one knew for the case $N_{\ell}=3$ 
that $\rho^{Q}$ contained complex eigenvalues, a natural normalization would be

$$
\rho^{Q}=\left[\begin{array}{ccc}
\rho_{11}^{Q} & 0 & 0 \\
\rho_{21}^{Q} & a & \rho_{23}^{Q} \\
\rho_{31}^{Q} & \rho_{32}^{Q} & a
\end{array}\right]
$$

with $\rho_{23}^{Q} \leq \rho_{32}^{Q}$ The value of $a$ is then uniquely pinned down by the real part of the complex eigenvalues. However, if the eigenvalues are all real, this is a more awkward form than the usual

$$
\rho^{Q}=\left[\begin{array}{ccc}
\rho_{11}^{Q} & 0 & 0 \\
\rho_{21}^{Q} & \rho_{22}^{Q} & 0 \\
\rho_{31}^{Q} & \rho_{32}^{Q} & \rho_{33}^{Q}
\end{array}\right]
$$

with $\rho_{11}^{Q} \geq \rho_{22}^{Q} \geq \rho_{33}^{Q}$. The estimation approach that we propose below will instantly reveal whether or not the lower triangular form (34) is imposing a restriction relative to the fullinformation maximum likelihood unrestricted values. If (34) is determined not to impose a restriction, one can feel confident in using the conventional parameterization, whereas if it does turn out to be inconsistent with the estimated unrestricted dynamics, the researcher should instead parameterize dynamics using (33). 


\subsection{Example 2: Macro finance model with single lag.}

We next examine the MF1 specification of Pericoli and Taboga (2008). Calculations similar to those for the latent factor model show the reduced form to be

$$
\begin{aligned}
\underset{\left(N_{m} \times 1\right)}{f_{t}^{m}} & =\underset{\left(N_{m} \times 1\right)}{A_{m}^{*}}+\underset{\left(N_{m} \times N_{m}\right)}{\phi_{m m}^{*}} f_{t-1}^{m}+\underset{\left(N_{m} \times N_{\ell}\right)}{\phi_{t-1}^{*}} Y_{m t}^{1}+u_{m t}^{*} \\
\underset{\left(N_{\ell} \times 1\right)}{Y_{t}^{1}} & =\underset{\left(N_{\ell} \times 1\right)}{A_{1}^{*}}+\underset{\left(N_{\ell} \times N_{m}\right)}{\phi_{1 m}^{*}} f_{t-1}^{m}+\underset{\left(N_{\ell} \times N_{\ell}\right)}{\phi_{11}^{*}} Y_{t-1}^{1}+\underset{\left(N_{\ell} \times N_{m}\right)}{\psi_{1 m}^{*}} f_{t}^{m}+u_{1 t}^{*} \\
\underset{\left(N_{e} \times 1\right)}{Y_{t}^{2}} & =\underset{\left(N_{e} \times 1\right)}{A_{2}^{*}}+\underset{\left(N_{e} \times N_{m}\right)}{\phi_{2 m}^{*}} f_{t}^{m}+\underset{\left(N_{e} \times N_{\ell}\right)}{\phi_{21}^{*}} Y_{t}^{1}+u_{2 t}^{*} .
\end{aligned}
$$

Once again it is convenient to include the contemporaneous value of $f_{t}^{m}$ in the equation for $Y_{t}^{1}$ and include contemporaneous values of both $f_{t}^{m}$ and $Y_{t}^{1}$ in the equation for $Y_{t}^{2}$ in order to orthogonalize the reduced-form residuals $u_{j t}^{*}$; the benefits of this representation will be seen in the next section. The mapping between structural and reduced-form parameters is given 
by the following equations and summarized in Table 3 with $N_{f}=N_{m}+N_{\ell}$ :

$$
\begin{aligned}
& A_{m}^{*}=c_{m}-\rho_{m \ell} B_{1 \ell}^{-1} A_{1} \\
& \phi_{m m}^{*}=\rho_{m m}-\rho_{m \ell} B_{1 \ell}^{-1} B_{1 m} \\
& \phi_{m 1}^{*}=\rho_{m \ell} B_{1 \ell}^{-1} \\
& A_{1}^{*}=A_{1}+B_{1 \ell} c_{\ell}-B_{1 \ell} \rho_{\ell \ell} B_{1 \ell}^{-1} A_{1} \\
& \phi_{1 m}^{*}=B_{1 \ell} \rho_{\ell m}-B_{1 \ell} \rho_{\ell \ell} B_{1 \ell}^{-1} B_{1 m} \\
& \phi_{11}^{*}=B_{1 \ell} \rho_{\ell \ell} B_{1 \ell}^{-1} \\
& \psi_{1 m}^{*}=B_{1 m} \\
& A_{2}^{*}=A_{2}-B_{2 \ell} B_{1 \ell}^{-1} A_{1} \\
& \phi_{2 m}^{*}=B_{2 m}-B_{2 \ell} B_{1 \ell}^{-1} B_{1 m} \\
& \phi_{21}^{*}=B_{2 \ell} B_{1 \ell}^{-1} \\
& \operatorname{Var}\left[\begin{array}{c}
u_{m t}^{*} \\
u_{1 t}^{*} \\
u_{2 t}^{*}
\end{array}\right]=\left[\begin{array}{ccc}
\Omega_{m}^{*} & 0 & 0 \\
0 & \Omega_{1}^{*} & 0 \\
0 & 0 & \Omega_{2}^{*}
\end{array}\right]=\left[\begin{array}{ccc}
\Sigma_{m m} \Sigma_{m m}^{\prime} & 0 & 0 \\
0 & B_{1 \ell} B_{1 \ell}^{\prime} & 0 \\
0 & 0 & \Sigma_{e} \Sigma_{e}^{\prime}
\end{array}\right]
\end{aligned}
$$

with $\Omega_{2}^{*}$ diagonal and $B_{1}$ and $B_{2}$ partitioned as described in Appendix A.

Once again inspection of the above equations reveals that the structure is unidentified. One can see this immediately for the case $N_{\ell}=3, N_{m}=2, N_{e}=3$ simply by counting parameters- there are 69 unknown structural parameters and only 66 reduced-form parameters from which they are supposed to be inferred. The problem arises in particular from the fact 
that, for the example we have been discussing, the observable implications of the 30 structural parameters in $\rho^{Q}$ and $\delta_{1}$ are completely captured by the 27 values of $\psi_{1 m}^{*}, \phi_{2 m}^{*}, \phi_{21}^{*}$, and $\Omega_{1}^{*}$. More fundamentally, the lack of identification would remain with this structure no matter how large the value of $N_{e}$. One can see this by verifying that the following transformation is perfectly allowed under the stated normalization but would not change the value of any reduced-form parameter: $B_{1 \ell} \rightarrow B_{1 \ell} H^{\prime}, c_{\ell} \rightarrow H c_{\ell}, \rho_{m \ell} \rightarrow \rho_{m \ell} H^{\prime}, \rho_{\ell \ell} \rightarrow H \rho_{\ell \ell} H^{\prime}, \rho_{\ell m} \rightarrow H \rho_{\ell m}$, and $B_{2 \ell} \rightarrow B_{2 \ell} H^{\prime}$, where $H$ could be any $\left(N_{\ell} \times N_{\ell}\right)$ orthogonal matrix.

There is also a separate identification problem arising from the fact that only maturities for which $n$ is an even number are included in the observation set. This means that only even powers of $\rho^{Q}$ appear in (15) and (16), which allows observationally equivalent sign transformations through $H$ as well.

\subsection{Example 3: Macro finance model with 12 lags.}

Last we consider the MF12 example, for which the reduced form is

$$
\begin{aligned}
\underset{(2 \times 1)}{f_{t}^{m}} & =\underset{(2 \times 24)}{\phi_{m m}^{*}} F_{t-1}^{m}+u_{m t}^{*} \\
\underset{(3 \times 1)}{Y_{t}^{1}} & =A_{1}^{*}+\underset{(3 \times 24)}{\phi_{1 m}^{*}} F_{t-1}^{m}+\underset{(3 \times 3)}{\phi_{11}^{*}} Y_{t-1}^{1}+\underset{(3 \times 2)}{\psi_{1 m}^{*}} f_{t}^{m}+u_{1 t}^{*} \\
\underset{(2 \times 1)}{Y_{t}^{2}} & =A_{2}^{*}+\underset{\substack{(2 \times 24) \\
\phi_{2 m}^{*}}}{m} F_{t}^{m}+\underset{(2 \times 3)}{\phi_{21}^{*}} Y_{t}^{1}+u_{2 t}^{*}
\end{aligned}
$$




$$
\begin{aligned}
& \phi_{m m}^{*}=\left[\begin{array}{llll}
\rho_{1} & \rho_{2} & \cdots & \rho_{12}
\end{array}\right] \\
& A_{1}^{*}=A_{1}-B_{1 \ell} \rho_{\ell \ell} B_{1 \ell}^{-1} A_{1} \\
& \underset{(3 \times 24)}{\phi_{1 m}^{*}}=\left[\begin{array}{cc}
B_{1 m}^{(1)} & 0 \\
(3 \times 22) & (3 \times 2)
\end{array}\right]-\underset{(3 \times 3)}{B_{1 \ell} \rho_{\ell \ell} B_{1 \ell}^{-1}}\left[\begin{array}{cc}
B_{1 m}^{(0)} & B_{1 m}^{(1)} \\
(3 \times 2) & (3 \times 22)
\end{array}\right] \\
& \phi_{11}^{*}=B_{1 \ell} \rho_{\ell \ell} B_{1 \ell}^{-1} \\
& \psi_{1 m}^{*}=B_{1 m}^{(0)} \\
& A_{2}^{*}=A_{2}-B_{2 \ell} B_{1 \ell}^{-1} A_{1} \\
& \phi_{2 m}^{*}=B_{2 m}-B_{2 \ell} B_{1 \ell}^{-1} B_{1 m} \\
& \phi_{21}^{*}=B_{2 \ell} B_{1 \ell}^{-1} \\
& \operatorname{Var}\left(\left[\begin{array}{c}
u_{m t}^{*} \\
u_{1 t}^{*} \\
u_{2 t}^{*}
\end{array}\right]\right)=\left[\begin{array}{ccc}
\Omega_{m}^{*} & 0 & 0 \\
0 & \Omega_{1}^{*} & 0 \\
0 & 0 & \Omega_{2}^{*}
\end{array}\right]=\left[\begin{array}{ccc}
\Sigma_{m m} \Sigma_{m m}^{\prime} & 0 & 0 \\
0 & B_{1 \ell} B_{1 \ell}^{\prime} & 0 \\
0 & 0 & \Sigma_{e} \Sigma_{e}^{\prime}
\end{array}\right]
\end{aligned}
$$

with $\Omega_{2}^{*}$ again diagonal and details on the partitioning of $B_{1}$ and $B_{2}$ in Appendix B. Table 4 summarizes the mapping between reduced-form and structural parameters. Note that the only reduced-form parameters relevant for inference about the 6 elements of $\delta_{0}$ and $\lambda$ are the 5 values for $A_{1}^{*}$ and $A_{2}^{*}$, establishing that these structural parameters are in fact unidentified. One might have thought that perhaps $\delta_{0}$ could be inferred separately from the OLS regression (23), freeing up the parameters $A_{1}^{*}$ and $A_{2}^{*}$ for estimation solely of $\lambda$. However, this is not the case, since the short-term interest rate is the same dependent variable in both regression (23) and in the first OLS regression from which $A_{1}^{*}$ is inferred. Another way to see this is to note that at most what one can expect to uncover from the 5 values of $A_{1}^{*}$ and $A_{2}^{*}$ are the 5 values of $A_{1}$ and $A_{2}$. The first element of $A_{1}$ is exactly equal to $\delta_{0}$, so even if $\delta_{0}$ were known a priori, 
the most that one could infer from $A_{1}$ and $A_{2}$ is 4 other parameters. Hence $A_{1}$ and $A_{2}$ would not be sufficient to uncover the 5 unknowns in $\lambda$ even if $\delta_{0}$ were known with certainty.

Ang and Piazzesi's (2003) Macro Model with its proposed identifying restrictions thus turns out to be unidentified at all points of the parameter space. In their empirical analysis, Ang and Piazzesi imposed an additional set of restrictions that were intended to improve estimation efficiency, though as we have just seen some of these are necessary for identification. We discuss these further in Section 4.4 below.

\section{Estimation.}

The reduced-form parameters are trivially obtained via OLS. Hence a very attractive alternative to numerical maximization of the log likelihood function directly with respect to the structural parameters $\theta$ is to let OLS do the work of maximizing the likelihood with respect to the reduced-form parameters, and then translate these into their implications for $\theta$. We demonstrate in this section how this can be done.

\subsection{Minimum-chi-square estimation.}

Let $\pi$ denote the vector consisting of reduced-form parameters (VAR coefficients and nonredundant elements of the variance matrices $), \mathcal{L}(\pi ; Y)$ denote the log likelihood for the entire sample, and $\hat{\pi}=\arg \max \mathcal{L}(\pi ; Y)$ denote the full-information-maximum-likelihood estimate. 
If $\hat{R}$ is a consistent estimate of the information matrix,

$$
R=-T^{-1} E\left[\frac{\partial^{2} \mathcal{L}(\pi ; Y)}{\partial \pi \partial \pi^{\prime}}\right]
$$

then we could test the hypothesis that $\pi=g(\theta)$ for $\theta$ a known vector of parameters by calculating the usual Wald statistic

$$
T[\hat{\pi}-g(\theta)]^{\prime} \hat{R}[\hat{\pi}-g(\theta)]
$$

which would have an asymptotic $\chi^{2}(q)$ distribution under the null hypothesis where $q$ is the dimension of $\pi$. Rothenberg (1973, p. 24) noted that one could also use (52) as a basis for estimation by choosing as an estimate $\hat{\theta}$ the value that minimizes this chi-square statistic.

Following Rothenberg (1973, pp. 24-25), we can obtain asymptotic standard errors by considering the linear approximation $g(\theta) \simeq \gamma+\Gamma \theta$ for $\Gamma=\partial g(\theta) /\left.\partial \theta^{\prime}\right|_{\theta=\theta_{0}}$ and $\gamma=g\left(\theta_{0}\right)-\Gamma \theta_{0}$ where $\hat{\pi} \stackrel{p}{\rightarrow} \pi_{0}$ and we assume there exists a value of $\theta_{0}$ for which the true model satisfies $g\left(\theta_{0}\right)=\pi_{0}$. Define the linearized minimum-chi-square estimator $\hat{\theta}^{*}$ as the solution to

$$
\min _{\theta} T[\hat{\pi}-\gamma-\Gamma \theta]^{\prime} R[\hat{\pi}-\gamma-\Gamma \theta]
$$

that is, $\hat{\theta}^{*}$ satisfies $\Gamma^{\prime} R\left(\hat{\pi}-\gamma-\Gamma \hat{\theta}^{*}\right)=0$ or $\hat{\theta}^{*}=\left(\Gamma^{\prime} R \Gamma\right)^{-1} \Gamma^{\prime} R(\hat{\pi}-\gamma)$. Since $\sqrt{T}(\hat{\pi}-$ $\left.\pi_{0}\right) \stackrel{L}{\rightarrow} N\left(0, R^{-1}\right)$, it follows that $\sqrt{T}\left(\hat{\theta}^{*}-\theta_{0}\right) \stackrel{L}{\rightarrow} N\left(0,\left[\Gamma^{\prime} R \Gamma\right]^{-1}\right)$. Hence our proposal is to approximate the variance of $\hat{\theta}$ with $T^{-1}\left(\hat{\Gamma}^{\prime} \hat{R} \hat{\Gamma}\right)^{-1}$ for $\hat{\Gamma}=\partial g(\theta) /\left.\partial \theta^{\prime}\right|_{\theta=\hat{\theta}}$.

We show in Appendix $\mathrm{E}$ that this is in fact identical to the usual asymptotic variance 
for the MLE as obtained from second derivatives of the log likelihood function directly with respect to $\theta$. In other words, the MCSE and MLE are asymptotically equivalent, and the MCSE inherits all the asymptotic optimality properties of the MLE. If in a particular sample the MCSE and MLE differ, there is no basis for claiming that one has better properties than the other.

In the case of a just-identified model, the minimum value attainable for (52) is zero, in which case one can without loss of generality simply minimize

$$
[\hat{\pi}-g(\theta)]^{\prime}[\hat{\pi}-g(\theta)]
$$

Note that in this case, if the optimized value for this objective is zero, then $\hat{\theta}$ is numerically identical to the value that achieves the global maximum of the likelihood written as a function of $\theta$. Although $\hat{\theta}_{M C S E}$ in this case is identical to $\hat{\theta}_{M L E}$, arriving at the estimate by the minimum-chi-square algorithm has two big advantages over the traditional brute-force maximization of the likelihood function. First, one knows instantly whether $\hat{\theta}$ corresponds to a global maximum of the original likelihood surface simply by checking whether a zero value is achieved for (53). By contrast, under the traditional approach, one has to try hundreds of starting values to be persuaded that a global maximum has been found, and even then cannot be sure. A second advantage is that minimization of (52) or (53) is far simpler computationally than brute-force maximization of the original likelihood function.

In addition, the greater computational ease makes calculation of small-sample confidence intervals feasible. The models considered here imply a reduced form that can be written in 
companion form as

$$
Y_{t}=k+\Phi Y_{t-1}+\Sigma_{Y} u_{t}
$$

for $Y_{t}$ the $(N \times 1)$ vector of observed variables (yields, macro variables, and possible lags of macro variables) and $u_{t} \sim N\left(0, I_{N}\right)$, where the parameters $k, \Phi$, and $\Sigma_{Y}$ are known functions of $\pi$. We can then obtain bootstrap confidence intervals for $\theta$ as follows. For artificial sample $j$, we will generate a sequence $\left\{u_{t}^{(j)}\right\}_{t=1}^{T}$ of $N\left(0, I_{N}\right)$ variables for $T$ the original sample size, and then recursively generate $Y_{t}^{(j)}=k(\hat{\pi})+\Phi(\hat{\pi}) Y_{t-1}^{(j)}+\Sigma_{Y}(\hat{\pi}) u_{t}^{(j)}$ for $t=1,2, \ldots, T$, starting from $Y_{0}^{(j)}=Y_{0}$, the initial value from the original sample, and using the identical parameter values $k, \Phi$, and $\Sigma_{Y}$ (as implied by the original $\hat{\pi}$ ) for each sample $j$. On sample $j$ we find the FIML estimate $\hat{\pi}^{(j)}$ on that artificial sample and then calculate $\hat{\theta}^{(j)}=\arg \min _{\theta} T\left[\hat{\pi}^{(j)}-g(\theta)\right]^{\prime} \hat{R}^{(j)}\left[\hat{\pi}^{(j)}-g(\theta)\right]$. We generate a sequence $j=1,2, \ldots, J$ of such samples, from which we could calculate $95 \%$ small-sample confidence intervals for each element of $\theta$. The small-sample standard errors for parameter $i$ reported in the following section were calculated from $\sqrt{J^{-1} \sum_{j=1}^{J}\left(\hat{\theta}_{i, M C S E}^{(j)}-\hat{\theta}_{i}\right)^{2}}$ where $\hat{\theta}_{i}$ is the MCSE estimate for the original sample (whose original FIML $\hat{\pi}$ was used to generate each artificial sample $j$ ) and $\hat{\theta}_{i, M C S E}^{(j)}$ is the minimum-chi-square estimate for artificial sample $j$.

We now illustrate these methods and their advantages in detail using the examples of affine term structure models discussed above.

\subsection{Example 1: Latent factor model.}

In the case of $N_{e}=1$, the latent factor model is just-identified, making application of minimum-chi-square estimation particularly attractive. The reduced-form parameter vec- 
tor here is

$$
\pi=\left(\left\{\operatorname{vec}\left(\left[\begin{array}{cc}
A_{1}^{*} & \phi_{11}^{*}
\end{array}\right]^{\prime}\right)\right\}^{\prime},\left[\operatorname{vech}\left(\Omega_{1}^{*}\right)\right]^{\prime},\left\{\operatorname{vec}\left(\left[\begin{array}{cc}
A_{2}^{*} & \phi_{21}^{*}
\end{array}\right]^{\prime}\right)\right\}^{\prime},\left[\operatorname{diag}\left(\Omega_{2}^{*}\right)\right]^{\prime}\right)^{\prime}
$$

where $\operatorname{vec}(X)$ stacks the columns of the matrix $X$ into a vector. If $X$ is square, $\operatorname{vech}(X)$ does the same using only the elements on or below the principal diagonal, and $\operatorname{diag}(X)$ constructs a vector from the diagonal elements of $X$. Because $u_{1 t}^{*}$ and $u_{2 t}^{*}$ are independent, full-informationmaximum-likelihood (FIML) estimation of $\pi$ is obtained by treating the $Y_{1}$ and $Y_{2}$ blocks separately. Since each equation of (24) has the same explanatory variables, FIML for the $i$ th row of $\left[A_{1}^{*}, \phi_{11}^{*}\right]$ is obtained by OLS regression of $Y_{i t}^{1}$ on a constant and $Y_{t-1}^{1}$, with $\hat{\Omega}_{1}^{*}$ the matrix of average outer products of those OLS residuals:

$$
\hat{\Omega}_{1}^{*}=T^{-1} \sum_{t=1}^{T}\left(Y_{t}^{1}-\hat{A}_{1}^{*}-\hat{\phi}_{11}^{*} Y_{t-1}^{1}\right)\left(Y_{t}^{1}-\hat{A}_{1}^{*}-\hat{\phi}_{11}^{*} Y_{t-1}^{1}\right)^{\prime}
$$

FIML estimates of the remaining elements of $\pi$ are likewise obtained from OLS regressions of $Y_{i t}^{2}$ on a constant and $Y_{t}^{1}$.

The specific mapping in Table 2 suggests that we can use the following multi-step algorithm to minimize (53) for the latent factor model with $N_{\ell}=3$ and $N_{e}=1$.

Step 1. The estimate of $\Sigma_{e}$ is obtained analytically from the square root of $\hat{\Omega}_{2}^{*}$.

Step 2. The estimates of the 9 unknowns in $\rho^{Q}$ and $\delta_{1}$ are found by numerically solving the 9 equations in (29) and (31)

$$
\left[B_{2}\left(\hat{\rho}^{Q}, \hat{\delta}_{1}\right)\right]\left[B_{1}\left(\hat{\rho}^{Q}, \hat{\delta}_{1}\right)\right]^{\prime}=\hat{\phi}_{21}^{*} \hat{\Omega}_{1}^{*}
$$




$$
\left[B_{1}\left(\hat{\rho}^{Q}, \hat{\delta}_{1}\right)\right]\left[B_{1}\left(\hat{\rho}^{Q}, \hat{\delta}_{1}\right)\right]^{\prime}=\hat{\Omega}_{1}^{*}
$$

Specifically, we do this by letting ${ }^{14} \hat{\pi}_{2}=\left(\left[\operatorname{vec}\left(\hat{\phi}_{21}^{*} \hat{\Omega}_{1}^{*}\right)\right]^{\prime},\left[\operatorname{vech}\left(\hat{\Omega}_{1}^{*}\right)\right]^{\prime}\right)^{\prime}$ and $g_{2}\left(\rho^{Q}, \delta_{1}\right)=\left(\left[\operatorname{vec}\left(B_{2} B_{1}^{\prime}\right)\right]^{\prime}\right.$, $\left.\left[\operatorname{vech}\left(B_{1} B_{1}^{\prime}\right)\right]^{\prime}\right)^{\prime}$ and finding $\hat{\rho}^{Q}$ and $\hat{\delta}_{1}$ by numerical minimization of $\left[\hat{\pi}_{2}-g_{2}\left(\rho^{Q}, \delta_{1}\right)\right]^{\prime}\left[\hat{\pi}_{2}-\right.$ $\left.g_{2}\left(\rho^{Q}, \delta_{1}\right)\right]$.

Step 3. The estimate of $\rho$ can then be obtained analytically from (26):

$$
\hat{\rho}=\hat{B}_{1}^{-1} \hat{\phi}_{11}^{*} \hat{B}_{1}
$$

where $\hat{B}_{1}$ is known from Step 2 .

Step 4. Numerically solve the 4 unknowns in $\delta_{0}$ and $c^{Q}$ from the 4 equations in $\hat{A}_{1}^{*}$ and $\hat{A}_{2}^{*}$ using (25) and (28):

$$
\begin{gathered}
\left(I_{3}-\hat{B}_{1} \hat{\rho} \hat{B}_{1}^{-1}\right) A_{1}\left(\delta_{0}, c^{Q}, \hat{\rho}^{Q}, \hat{\delta}_{1}\right)=\hat{A}_{1}^{*} \\
A_{2}\left(\delta_{0}, c^{Q}, \hat{\rho}^{Q}, \hat{\delta}_{1}\right)-\hat{B}_{2} \hat{B}_{1}^{-1} A_{1}\left(\delta_{0}, c^{Q}, \hat{\rho}^{Q}, \hat{\delta}_{1}\right)=\hat{A}_{2}^{*} .
\end{gathered}
$$

Although Steps 2 and 4 involve numerical minimization, these are computationally far simpler problems than that associated with traditional brute-force maximization of the likelihood function with respect to the full vector $\theta$. To illustrate this, we repeated the experiment described in Section 2.2 with the same 100 starting values. Whereas we saw in Section 2.2

\footnotetext{
${ }^{14}$ To assist with scaling for numerical robustness, we multiplied each equation in step 2 by $1200 \times 1$.e +7 and those in step 4 below by $1 . e+8$. If we were minimizing (52) directly one would automatically achieve optimal scaling by using $\hat{R}$ in place of a constant $k$ times the identity matrix as here. However, our formulation takes advantage of the fact that the elements of $\hat{\pi}$ can be rearranged in order to avoid inversion of $B_{1}$ inside the numerical optimization, in which case $\hat{R}$ is no longer the optimal weighting matrix. The minimization was implemented using the fsolve command in MATLAB. We also multiplied $\delta_{1}$ by 1000 to improve numerical robustness.
} 
that only one of these efforts found the global maximum under the traditional approach, with our method all 100 converge to the global MLE in one of the 6 configurations that are observationally equivalent for the original normalization. One of the reasons for the greater robustness is that the critical stumbling block for the traditional method- numerical search over $\rho$ - is completely avoided since in our approach (54) is solved analytically. Another is that $c^{Q}$ and uncertainties about its scale are completely eliminated from the core problem of estimation of $\rho^{Q}$ and $\delta_{1}$

Joslin, Singleton, and Zhu (2011) have recently proposed a promising alternative parameterization of the pure latent affine models that shares some of the advantages of our approach. They parameterize the system such that $A_{1}^{*}$ and $\phi_{11}^{*}$ in (24) are taken to be the direct objects of interest, and as in our approach, estimate these directly with OLS. But whereas our approach also uses the OLS estimates of $A_{2}^{*}$ and $\phi_{21}^{*}$ in (27) to uncover the remaining affinepricing parameters, their approach finds these by maximizing the joint likelihood function of $Y_{1}$ and $Y_{2}$. Although they report that the second step involves no numerical difficulties, our experience is that while it offers a significant improvement over the traditional method, it is still susceptible to some of the same problems. For example, we repeated the experiment described above with the same data set and same starting values for $\delta_{0}$ and the 3 unknown diagonal elements in $\rho^{Q}$ that appear in their parameterization as we used in the simulations described above, starting the search for $\Omega_{1}^{*}$ from the OLS estimates as they recommend. We found that the algorithm found the global maximum in 54 out of the 100 trials $^{15}$, but got stuck in regions with diagonal elements of $\rho^{Q}$ equal to unity in the others, in a similar failure

\footnotetext{
${ }^{15}$ To assist the numerical search, we multiplied $\Omega_{1}^{*}$ by 1000 . Without this scaling, the searches only succeeded in finding the global maximum in 14 of the 100 trials.
} 
of local identification that we documented above can plague the traditional approach.

We applied our method directly to the Ang and Piazzesi interest rate data described in more detail in Section 4.4 below. Table 5 reports the resulting minimum-chi-square estimates (identical in this case to the full-information-maximum-likelihood estimates). The table also reports asymptotic standard errors in parentheses and small-sample standard errors in square brackets. The latter were calculated by applying our method to each of 1000 separate data sets, each generated from the vector autoregression estimated from the original data set. Note that the fact that we can verify with certainty that the global maximum has been found on each of these 1000 simulated data sets is part of what makes calculation of small-sample standard errors feasible and attractive. Finding the FIML estimate on 1000 data sets takes about 90 seconds on a PC. For this example, we find that the asymptotic standard errors provide an excellent approximation to the true small-sample values.

Although our original inference was conducted in terms of a $Q$ representation, we report the implied $\lambda$ representation values in the right-hand columns of Table 5, since that is the form in which parameter estimates are often reported for these models. Our suggestion is that the approach we illustrate here, of beginning with a completely unrestricted model to see which parameters appear to be most significant, has many advantages over the traditional approach $^{16}$ in which sundry restrictions are imposed at a very early stage, partly in order to assist with identification and estimation.

\footnotetext{
${ }^{16}$ See for example Duffee (2002) and Duarte (2004).
} 


\subsection{Example 2: Macro finance model with single lag.}

We also applied this procedure to estimate parameters for our MF1 example using a slightly different quarterly data set from Pericoli and Taboga. We used constant-maturity Treasury yields as of the first day of the quarter, dividing the numbers as usually reported by 400 in order to convert to units of quarterly yield on which formulas such as (14) are based. We estimated inflation from the 12-month percentage change in the CPI and the output gap by applying the Hodrick-Prescott filter with $\lambda=1600$ to 100 times the natural log of real GDP. Data run from 1960:Q1 to 2007:Q1 and were obtained from the FRED database of the Federal Reserve Bank of St. Louis.

If we impose 3 further restrictions on $\rho_{\ell \ell}^{Q}$ relative to the original formulation, the MF1 model presented above would be just-identified in terms of parameter count, for which we would logically again simply try to invert the reduced-form parameter estimates to obtain the FIML estimates of the structural parameters. Once again orthogonality of the residuals across the three blocks of (35) through (37) means FIML estimation can be done on each block separately, and within each block implemented by OLS equation by equation. Our estimation procedure on this system is then as follows.

Step 1. The $f_{t}^{m}$ and $Y_{t}^{2}$ variance parameters are obtained analytically from (48), that is, $\hat{\Sigma}_{m m}$ from the Cholesky factorization of $\hat{\Omega}_{m}^{*}$ and $\hat{\Sigma}_{e}$ from the square root of $\hat{\Omega}_{2}^{*}$.

Step 2. Using (44), (46), (48), and (47), choose the values of $\rho^{Q}$ and $\delta_{1}$ so as to solve the 
following equations numerically ${ }^{17}$ :

$$
\begin{gathered}
B_{1 m}\left(\rho^{Q}, \delta_{1}\right)=\hat{\psi}_{1 m}^{*} \\
B_{2 m}\left(\rho^{Q}, \delta_{1}\right)=\hat{\phi}_{2 m}^{*}+\hat{\phi}_{21}^{*} \hat{\psi}_{1 m}^{*} \\
\operatorname{vech}\left\{\left[B_{1 \ell}\left(\rho^{Q}, \delta_{1}\right)\right]\left[B_{1 \ell}\left(\rho^{Q}, \delta_{1}\right)\right]^{\prime}\right\}=\operatorname{vech}\left(\hat{\Omega}_{1}^{*}\right) \\
{\left[B_{2 \ell}\left(\rho^{Q}, \delta_{1}\right)\right]\left[B_{1 \ell}\left(\rho^{Q}, \delta_{1}\right)\right]^{\prime}=\hat{\phi}_{21}^{*} \hat{\Omega}_{1}^{*} .}
\end{gathered}
$$

We initially tried to solve this system for $\rho_{\ell \ell}^{Q}$ of the lower-triangular form (34), but found no solution exists, indicating that the FIML estimate of $\rho_{\ell \ell}^{Q}$ has complex roots. We accordingly reparameterized $\rho_{\ell \ell}^{Q}$ in the form (33), for which an exact solution was readily obtained.

Step 3. From these estimates one then analytically can calculate $\hat{\rho}_{m \ell}, \hat{\rho}_{m m}, \hat{\rho}_{\ell \ell}$, and $\hat{\rho}_{\ell m}$ from $\hat{\phi}_{m 1}^{*}, \hat{\phi}_{m m}^{*}, \hat{\phi}_{11}^{*}$, and $\hat{\phi}_{1 m}^{*}$, respectively.

Step 4. Since $c_{m}$ and $c_{\ell}$ are unrestricted, the values of $\delta_{0}$ and $c^{Q}$ can be inferred solely from $A_{2}^{*}$ by numerical solution of (45):

$$
A_{2}\left(\delta_{0}, c^{Q}, \hat{\rho}^{Q}, \hat{\delta}_{1}\right)-\hat{B}_{2 \ell} \hat{B}_{1 \ell}^{-1} \hat{A}_{1}\left(\delta_{0}, c^{Q}, \hat{\rho}^{Q}, \hat{\delta}_{1}\right)=\hat{A}_{2}^{*} .
$$

Step 5. We then can calculate the remaining parameters analytically using (38) and (41):

$$
\hat{c}_{m}=\hat{A}_{m}^{*}+\hat{\rho}_{m \ell} \hat{B}_{1 \ell}^{-1} \hat{A}_{1}
$$

\footnotetext{
${ }^{17}$ To improve accuracy of the numerical algorithm, we multiplied the last two equations by 400 and then the whole set of equations by 1.e+7. The parameter $\delta_{1}$ was also scaled by 100 .
} 


$$
\hat{c}_{\ell}=\hat{B}_{1 \ell}^{-1}\left(\hat{A}_{1}^{*}-\hat{A}_{1}+\hat{B}_{1 \ell} \hat{\rho}_{\ell \ell} \hat{B}_{1 \ell}^{-1} \hat{A}_{1}\right)
$$

Table 6 reports the FIML estimates obtained by the above algorithm along with asymptotic standard errors. These estimates would cause one to be cautious about the proposed modelstandard errors are quite large, and 3 eigenvalues of the estimated $\rho^{Q}$ matrix are outside the unit circle. We found small-sample standard errors much more difficult to calculate for this example, in part because the value of $\rho^{Q}$ associated with a given $\hat{\pi}^{(j)}$ can have anywhere from zero to four complex eigenvalues, with eigenvalues of the $\rho_{\ell \ell}^{Q}$ submatrix sometimes greater than 2 in modulus. Our interpretation is that further restrictions on the interaction between the macro and latent factors could be helpful for this class of models.

\subsection{Example 3. Macro finance model with 12 lags.}

Here our data set follows Ang and Piazzesi (2003) as closely as possible, using zero-coupon bond yields with maturities of 1, 3, 12, 36 and 60 months from CRSP monthly treasury file, each divided by 1200 to quote as monthly fractional rates. We obtained two groups of monthly US macroeconomic key indicators, seasonally adjusted if applicable, from Datastream. The first group consists of various inflation measures which are based on the CPI, the PPI of finished goods, and the CRB Spot Index for commodity prices. The second group contains variables that capture real activity: the Index of Help Wanted Advertising, Unemployment Rates, the growth rate of Total Civilian Employment and the growth rate of Industrial Production. All growth rates and inflation rates are measured as the difference in logs of the monthly index value between dates $t$ and $t-12$. We first normalized each series separately to have zero mean 
and unit variance, then extracted the first principal component of each group, designated the "inflation" and "real activity" indices, respectively, with each index having zero mean and unit variance by construction. The sample period for yields is from December 1952 to December 2000, and that for the macro indices is from January 1952 to December 2000. We assume that 1-, 12- and 60-month yields are priced exactly, and 3- and 36-month yields are priced with error $\left(N_{e}=2\right)$. We use the Ang and Piazzesi (2003) Macro Model with their additional proposed zero restrictions to illustrate minimum-chi-square estimation for an overidentified model.

The reduced-form equations (49)-(51) form 3 independent blocks. If we interpret $Y_{t}^{m}=$ $f_{t}^{m}$, we can write the structure of block $i$ for $i=1,2, m$ as

$$
\begin{gathered}
\underset{\left(q_{i} \times 1\right)}{Y_{t}^{i}}=\underset{\left(q_{i} \times k_{i}\right)\left(k_{i} \times 1\right)}{\prod_{i t}^{\prime}}+\underset{\left(q_{i} \times 1\right)}{u_{i t}^{*}} \\
u_{i t}^{*} \sim N\left(0, \Omega_{i}^{*}\right) .
\end{gathered}
$$

The information matrix for the full system of reduced-form parameters is

$$
\hat{R}=\left[\begin{array}{ccc}
\hat{R}_{m} & 0 & 0 \\
0 & \hat{R}_{1} & 0 \\
0 & 0 & \hat{R}_{2}
\end{array}\right]
$$


where as in Magnus and Neudecker (1988, p. 321)

$$
\hat{R}_{i}=\left[\begin{array}{cc}
\hat{\Omega}_{i}^{*-1} \otimes T^{-1} \sum_{t=1}^{T} x_{i t} x_{i t}^{\prime} & 0 \\
0 & (1 / 2) D_{q_{i}}^{\prime}\left(\hat{\Omega}_{i}^{*-1} \otimes \hat{\Omega}_{i}^{*-1}\right) D_{q_{i}}
\end{array}\right]
$$

for $D_{N}$ the $N^{2} \times N(N+1) / 2$ duplication matrix satisfying $D_{N} \operatorname{vech}(\Omega)=\operatorname{vec}(\Omega)$.

The structural parameters $\Sigma_{e}$ appear only in the last half of the third block, no other parameters appear in this block, and these 2 structural parameters are just-identified by the 2 diagonal elements of $\Omega_{2}^{*}$. Thus the minimum-chi-square estimates of $\Sigma_{e}$ are obtained immediately from the square roots of diagonal elements of $\hat{\Omega}_{2}^{*}$. The structural parameters $\rho_{1}, \ldots, \rho_{12}$ appear directly in the first block and, through $\rho^{Q}$, in the second and third blocks as well, so FIML or minimum-chi-square estimation would exploit this. However, to reduce dimensionality, we follow Ang and Piazzesi in replacing $\rho_{2}, \ldots, \rho_{12}$ where they appear in $\rho^{Q}$ with the OLS estimates $\hat{\rho}_{2}, \ldots, \hat{\rho}_{12}$. In order to try to replicate their setting as closely as possible, we also follow their procedure of imposing $\hat{\delta}_{1 m}$ on the basis of OLS estimation of (23). Hence the minimum-chi-square analog to their problem is to minimize an expression of the form of (52) with

$$
\begin{gathered}
\hat{\pi}=\left(\left[\operatorname{vec}\left(\hat{\Pi}_{1}\right)\right]^{\prime},\left[\operatorname{vech}\left(\hat{\Omega}_{1}^{*}\right)\right]^{\prime},\left[\operatorname{vec}\left(\hat{\Pi}_{2}\right)\right]^{\prime}\right)^{\prime} \\
\hat{R}=\left[\begin{array}{ccc}
\hat{\Omega}_{1}^{*-1} \otimes T^{-1} \sum_{t=1}^{T} x_{1 t} x_{1 t}^{\prime} & 0 \\
0 & (1 / 2) D_{3}^{\prime}\left(\hat{\Omega}_{1}^{*-1} \otimes \hat{\Omega}_{1}^{*-1}\right) D_{3} & 0 \\
0 & 0 & \hat{\Omega}_{2}^{*-1} \otimes T^{-1} \sum_{t=1}^{T} x_{2 t} x_{2 t}^{\prime}
\end{array}\right] \\
x_{1 t}=\left(1, F_{t-1}^{m \prime}, Y_{t-1}^{1 \prime}, f_{t}^{m \prime}\right)^{\prime}
\end{gathered}
$$




$$
\begin{aligned}
& x_{2 t}=\left(1, F_{t}^{m \prime}, Y_{t}^{1 \prime}\right)^{\prime} \\
& \hat{\Pi}_{i}^{\prime}=\left(\sum_{t=1}^{T} Y_{t}^{i} x_{i t}^{\prime}\right)\left(\sum_{t=1}^{T} x_{i t} x_{i t}^{\prime}\right)^{-1} \quad \text { for } i=1,2 \\
& \hat{\Omega}_{1}^{*}=T^{-1} \sum_{t=1}^{T}\left(Y_{t}^{1}-\hat{\Pi}_{1}^{\prime} x_{1 t}\right)\left(Y_{t}^{1}-\hat{\Pi}_{1}^{\prime} x_{1 t}\right)^{\prime} \\
& \hat{\Omega}_{2}^{*}=T^{-1} \sum_{t=1}^{T}\left[\begin{array}{ccc}
{\left[\hat{u}_{2 t}(1)\right]^{2}} & \cdots & 0 \\
\vdots & \vdots & \vdots \\
0 & \cdots & {\left[\hat{u}_{2 t}\left(N_{e}\right)\right]^{2}}
\end{array}\right]
\end{aligned}
$$

with $\hat{u}_{2 t}(j)$ the $j$ th element of $Y_{t}^{2}-\hat{\Pi}_{2}^{\prime} x_{2 t}$.

Ang and Piazzesi also imposed a further set of restrictions on parameters, setting parameters with large standard errors as estimated in their first stage to zero. Their understanding was that the purpose of these restrictions was to improve efficiency, though we saw in Section 3.3 that some of these restrictions are in fact necessary in order to achieve identification. Our purpose here is to illustrate the minimum-chi-square method on an overidentified structure, and we therefore attempt to estimate their final proposed structure using our method. The additional parameters that Ang and Piazzesi fixed at zero include the $(2,1)$ and $(3,1)$ elements of $\rho_{\ell \ell}$ (which recall was already lower triangular), the $(1,2),(2,2),(3,2)$ and $(1,3)$ elements of $\Lambda_{\ell \ell}$, both elements in $\lambda_{m}$, and the $2^{\text {nd }}$ and $3^{\text {rd }}$ elements of $\lambda_{\ell}$. Our goal is then to minimize (52) with respect to the 17 remaining unknown parameters, 1 in $\lambda_{\ell}, 4$ in $\Lambda_{m m}, 5$ in $\Lambda_{\ell \ell}, 4$ in $\rho_{\ell \ell}$, and 3 in $\delta_{1 \ell} \cdot{ }^{18}$

\footnotetext{
18 We made one other slight change in parameterization that may be helpful. Since $\Lambda_{\ell \ell}$ always enters either the minimum-chi-squared calculations or the original maximum likelihood estimation in the form of high powers of the matrix $\rho_{\ell \ell}^{Q}=\rho_{\ell \ell}-\Lambda_{\ell \ell}$, the algorithms will be better behaved numerically if the unknown elements of $\rho_{\ell \ell}^{Q}$ rather than those of $\Lambda_{\ell \ell}$ are taken to be the object of interest. Specifically, for this example
} 
The results of this estimation for 100 different starting values are reported in Table 7 . Our procedure uncovered three local minima to the objective function. The parameters we report as Local1 correspond to the values reported in Table 6 of Ang and Piazzesi. The small differences between our estimates and theirs are due to some slight differences between the data sets and the fact that, in an overidentified structure, the minimum-chi-square and maximum-likelihood estimates are not numerically identical. Our procedure establishes that the estimates reported by Ang and Piazzesi in fact represent only a local maximum of the likelihood- both the estimates we report as Local2 and Global achieve substantially higher values for the log likelihood function relative to Local1. Moreover, the differences between estimates in terms of the pricing of risk are substantial. In the original reported Ang and Piazzesi estimates, an increase in inflation lowers the price of inflation risk and raises the price of output risk, whereas the values implied by Global reverse these signs. This is consistent with their finding that the prices of observable macro risk behave very differently between their Macro Model and Macro Lag Model specifications-- we find they also differ substantially across alternative local maxima of the log likelihood function even within their single Macro Model specification. Note that the large prices of risk for these higher local maxima can make them easy to miss with conventional estimation and conventional starting values of zero price of risk.

Another benefit of the minimum-chi-square estimation is that the value for the objective we implemented this subject to the proposed restrictions by parameterizing

$$
\rho_{\ell \ell}=\left[\begin{array}{ccc}
\theta_{1} & 0 & 0 \\
0 & \theta_{2} & 0 \\
0 & \theta_{3} & \theta_{4}
\end{array}\right] \quad \rho_{\ell \ell}^{Q}=\left[\begin{array}{ccc}
\theta_{5} & 0 & 0 \\
\theta_{6} & \theta_{2} & \theta_{7} \\
\theta_{8} & \theta_{3} & \theta_{9}
\end{array}\right],
$$

and then translated back in terms of the implied values for $\Lambda_{\ell \ell}$ for purposes of reporting values in Table 7 . 
function itself gives us an immediate test of the various overidentifying restrictions. There are 152 parameters in the reduced form vector $\pi$ in (55). The 17 estimated elements of $\theta$ then leave 135 degrees of freedom. The $1 \%$ critical value for a $\chi^{2}(135)$ variable is 176 . Thus the observed minimum value for our objective function (462.15) provides overwhelming evidence that the restrictions imposed by the model are inconsistent with the observed data.

\section{Conclusion.}

There are considerable benefits from describing affine term structure models in terms of their implications for the reduced-form representation of the data, which for a popular class of models is simply a restricted Gaussian vector autoregression. In this paper we used this representation to develop an approach to characterizing identification that has not previously been used for affine term structure models. We demonstrated that three popular canonical representations are in fact not identified, and showed how convergence to an unidentified region of the parameter space can complicate numerical search. A second and separate contribution of the paper was to propose inferring structural parameters from the unrestricted OLS estimates by the method of minimum-chi-square estimation, which is an approach to parameter estimation that again has not previously been used for affine term structure models. We demonstrated that among other benefits, this method is asymptotically equivalent to maximum likelihood estimation and can in some cases make it feasible to calculate smallsample standard errors, to know instantly whether estimates represent a global or only a local optimum, and to recognize whether a given structure is unreasonably restricting the class of 
possible models.

By missing these insights, previous researchers have instead often imposed arbitrary restrictions in order to obtain estimates and in other cases failed to find the true global maximum of the likelihood function. By showing how to recognize an unidentified structure, greatly reducing the computational burden of estimation, and providing an immediate specification test of any proposed restrictions, we hope that our methods will help to make these models a more effective tool for research in macroeconomics and finance. 


\section{References}

Aït-Sahalia, Yacine, and Robert L. Kimmel, 2010, Estimating Affine Multifactor Term Structure Models Using Closed-Form Likelihood Expansions. Journal of Financial Economics, 98.

Ang, Andrew, Sen Dong, and Monika Piazzesi, 2007, No-Arbitrage Taylor Rules. National Bureau of Economic Research, Working paper no. 13448.

Ang, Andrew, and Monika Piazzesi, 2003, A No-Arbitrage Vector Autoregression of Term Structure Dynamics with Macroeconomic and Latent Variables. Journal of Monetary Economics, 50, 745-787.

Ang, Andrew, Monika Piazzesi, and Min Wei, 2006, What Does the Yield Curve Tell Us About GDP Growth. Journal of Econometrics, 131, 359-403.

Bauer, Michael D., 2011, Term Premia and the News. Federal Reserve Bank of San Francisco, Working paper.

Beechey, Meredith J., and Jonathan H. Wright, 2009, The High-Frequency Impact of News On Long-Term Yields and Forward Rates: Is It Real? Journal of Monetary Economics, 56, $535-544$.

Bekaert, Geert, Seonghoon Cho, and Antonio Moreno, 2010, New Keynesian Macroeconomics and the Term Structure. Journal of Money, Credit, and Banking, 42, 33-62.

Chamberlain, Gary, 1982, Multivariate Models for Panel Data. Journal of Econometrics, 18, $5-46$. 
Chen, Ren-Raw, and Louis Scott, 1993, Maximum Likelihood Estimation for a Multifactor Equilibrium Model of the Term Structure of Interest Rates. The Journal of Fixed Income, $3,14-31$.

Christensen, Jens H. E., Francis X. Diebold, and Glenn D. Rudebusch, 2011, The Affine Arbitrage-Free Class of Nelson-Siegel Term Structure Models. Journal of Econometrics, $164,4-20$.

Christensen, Jens H. E., Jose A. Lopez, and Glenn D. Rudebusch, 2009, Do Central Bank Liquidity Facilities Affect Interbank Lending Rates? Working paper 2009-13, Federal Reserve Bank of San Francisco.

Christensen, Jens H. E., Jose A. Lopez, and Glenn D. Rudebusch, 2010, Inflation Expectations and Risk Premiums in an Arbitrage-Free Model of Nominal and Real Bond Yields. Journal of Money, Credit, and Banking, 42, 143 - 178.

Cochrane, John H., and Monika Piazzesi, 2009, Decomposing the Yield Curve. AFA 2010 Atlanta Meetings Paper.

Collin-Dufresne, Pierre, Robert S. Goldstein, and Christopher S. Jones, 2008, Identification of Maximal Affine Term Structure Models. Journal of Finance, 63, 743-795.

Dai, Qiang, and Kenneth J. Singleton, 2000, Specification Analysis of Affine Term Structure Models. The Journal of Finance, 55, 1943-1978.

Dai, Qiang, and Kenneth J. Singleton, 2002, Expectation Puzzles, Time-Varying Risk Premia, and Affine Models of the Term Structure. Journal of Financial Economics, 63, 415-441. 
Duarte, Jefferson, 2004, Evaluating an Alternative Risk Preference in Affine Term Structure Models. Review of Financial Studies, 17, 379-404.

Duffee, Gregory R., 2002, Term Premia and Interest Rate Forecasts in Affine Models. The Journal of Finance, 57, 405-443.

Duffee, Gregory R., 2011, Forecasting with the Term Structure: The Role of No-Arbitrage Restrictions. Working Paper, Johns Hopkins University.

Duffee, Gregory R., and Richard H. Stanton, 2008, Evidence on Simulation Inference for Near Unit-Root Processes with Implications for Term Structure Estimation. Journal of Financial Econometrics, 6, $108-142$.

Duffie, Darrell, and Rui Kan, 1996, A Yield-Factor Model of Interest Rates. Mathematical Finance, 6, 379-406.

Fisher, Franklin M., 1966, The Identification Problem in Econometrics. New York: McGrawHill.

Fisher, R.A., 1924, The Conditions Under Which $\chi^{2}$ Measures the Discrepancey Between Observation and Hypothesis. Journal of the Royal Statistical Society, 87, $442-450$.

Gallant, A. Ronald, and George E. Tauchen, 1992, A Nonparametric Approach to Nonlinear Time Series Analysis: Estimation and Simulation. In New Directions in Time Series Analysis Part II, edited by David Brillinger, Peter Caines, John Geweke, Emanuel Parzen, Murray Rosenblatt, and Murad S. Taqqu, Springer-Verlag. 
Gourieroux, Christian, Alain Monfort, and Eric Renault, 1993, Indirect Inference. Journal of Applied Econometrics, 8S, S85 - S118.

Hamilton, James D., and Jing Cynthia Wu, forthcominga, The Effectiveness of Alternative Monetary Policy Tools in a Zero Lower Bound Environment. Journal of Money, Credit \& Banking.

Hamilton, James D., and Jing Cynthia Wu, forthcomingb, Testable Implications of Affine Term Structure Models. Journal of Econometrics.

Hansen, Lars P., 1982, Large Sample Properties of Generalized Method of Moments Estimators. Econometrica, 50, 1029 - 1054.

Hördahl, Peter, Oreste Tristani, and David Vestin, 2006, A Joint Econometric Model of Macroeconomic and Term-Structure Dynamics. Journal of Econometrics, 131, 405 - 444.

Joslin, Scott, Kenneth J. Singleton, and Haoxiang Zhu, 2011, A New Perspective On Gaussian Dynamic Term Structure Models. Review of Financial Studies, 24, 926-970.

Kim, Don H., 2008, Challenges in Macro-Finance Modeling. BIS Working Paper No. 240, FEDS Working Paper No. 2008-06.

Kim, Don H., and Athanasios Orphanides, 2005, Term Structure Estimation with Survey Data On Interest Rate Forecasts. Federal Reserve Board, Finance and Economics Discussion Series 2005-48.

Kim, Don H., and Jonathan H. Wright, 2005, An Arbitrage-Free Three-Factor Term Structure 
Model and the Recent Behavior of Long-Term Yields and Distant-Horizon Forward Rates.

Federal Reserve Board, Finance and Economics Discussion Series 2005-33.

Magnus, Jan R., and Heinz Neudecker, 1988, Matrix Differential Calculus with Applications in Statistics and Econometrics. John Wiley \& Sons, Ltd.

Malinvaud, Edmond, 1970, Statistical Methods of Econometrics, Third Revised Edition. New York: North-Holland.

Newey, Whitney K., 1987, Efficient Estimation of Limited Dependent Variable Models with Endogenous Explanatory Variables. Journal of Econometrics, 36, 231-250.

Neyman, J., and E. S. Pearson, 1928, On the Use and Interpretation of Certain Test Criteria for Purposes of Statistical Inference: Part II. Biometrika, 20A, 263 - 294.

Pericoli, Marcello, and Marco Taboga, 2008, Canonical Term-Structure Models with Observable Factors and the Dynamics of Bond Risk Premia. Journal of Money, Credit and Banking, 40, 1471-1488.

Rothenberg, Thomas J., 1971, Identification in Parametric Models. Econometrica, 39, 577591.

Rothenberg, Thomas J., 1973, Efficient Estimation with A Priori Information. Yale University Press.

Rudebusch, Glenn D., Eric T. Swanson, and Tao Wu, 2006, The Bond Yield 'Conundrum' From a Macro-Finance Perspective. Monetary and Economic Studies (Special Edition), 83128. 
Rudebusch, Glenn D., and Tao Wu, 2008, A Macro-Finance Model of the Term Structure, Monetary Policy and the Economy. The Economic Journal, 118, 906-926.

Singleton, Kenneth J., 2006, Empirical Dynamic Asset Pricing. Princeton University Press.

Smith, Jr., Anthony A., 1993, Estimating Nonlinear Time-Series Models Using Simulated Vector Autoregressions. Journal of Applied Econometrics, 8S, S63 - S84.

Smith, Josephine M., 2010, The Term Structure of Money Market Spreads During the Financial Crisis. Ph.D. thesis, Stanford University.

Vasicek, Oldrich, 1977, An Equilibrium Characterization of the Term Structure. Journal of Financial Economics, 5, 177-188. 


\section{Appendix A. Log likelihood function for the MF1 specification.}

The coefficients relating $Y_{t}^{1}$ and $Y_{t}^{2}$ to macro and latent factors can be partitioned as

$$
\left[\begin{array}{cc}
B_{1 m} & B_{1 \ell} \\
(3 \times 2) & (3 \times 3) \\
B_{2 m} & B_{2 \ell} \\
(3 \times 2) & (3 \times 3)
\end{array}\right]=\left[\begin{array}{c}
b_{4}^{\prime} \\
b_{20}^{\prime} \\
b_{40}^{\prime} \\
b_{8}^{\prime} \\
b_{12}^{\prime} \\
b_{28}^{\prime}
\end{array}\right]
$$

for $b_{n}$ given by (15). The conditional density for the th observation is then

$$
f\left(f_{t}^{m}, Y_{t} \mid f_{t-1}^{m}, Y_{t-1}\right)=\frac{1}{|\operatorname{det}(J)|} f\left(f_{t}^{m}, f_{t}^{\ell}, u_{t}^{e} \mid f_{t-1}^{m}, f_{t-1}^{\ell}, u_{t-1}^{e}\right)
$$

where

$$
\begin{gathered}
f\left(f_{t}^{m}, f_{t}^{\ell}, u_{t}^{e} \mid f_{t-1}^{m}, f_{t-1}^{\ell}, u_{t-1}^{e}\right)=f\left(f_{t}^{m} \mid f_{t-1}^{\ell}, f_{t-1}^{m}\right) f\left(f_{t}^{\ell}, \mid f_{t-1}^{\ell}, f_{t-1}^{m}\right) f\left(u_{t}^{e}\right) \\
f\left(f_{t}^{m} \mid f_{t-1}^{\ell}, f_{t-1}^{m}\right)=\phi\left(f_{t}^{m} ; c_{m}+\rho_{m m} f_{t-1}^{m}+\rho_{m \ell} f_{t-1}^{\ell}, \Sigma_{m m} \Sigma_{m m}^{\prime}\right) \\
f\left(f_{t}^{\ell} \mid f_{t-1}^{\ell}, f_{t-1}^{m}\right)=\phi\left(f_{t}^{\ell} ; c_{\ell}+\rho_{\ell m} f_{t-1}^{m}+\rho_{\ell \ell} f_{t-1}^{\ell}, I_{N_{\ell}}\right) \\
f\left(u_{t}^{e}\right)=\phi\left(u_{t}^{e} ; 0, I_{N_{e}}\right) \\
f_{t}^{\ell}=B_{1 \ell}^{-1}\left(Y_{t}^{1}-A_{1}-B_{1 m} f_{t}^{m}\right) \\
u_{t}^{e}=\Sigma_{e}^{-1}\left(Y_{t}^{2}-A_{2}-B_{2 m} f_{t}^{m}-B_{2 \ell} f_{t}^{\ell}\right) \\
J=\left[\begin{array}{cc}
B_{1 \ell} & 0 \\
B_{2 \ell} & \Sigma_{e}
\end{array}\right]
\end{gathered}
$$

For the $Q$ representation and our $N_{\ell}=3, N_{m}=2, N_{e}=3$ example, there are 25 unknown elements in $\rho, 25$ in $\rho^{Q}, 5$ in $c, 2$ in $c^{Q}, 5$ in $\delta_{1}, 1$ in $\delta_{0}, 3$ in $\Sigma_{m m}$, and 3 in $\Sigma_{e}$. The traditional approach is to arrive at estimates of these 69 parameters by numerical maximization of

$$
\mathcal{L}(\theta ; Y)=\sum_{t=1}^{T} \log f\left(f_{t}^{m}, Y_{t} \mid f_{t-1}^{m}, Y_{t-1}\right)
$$

as calculated using the above formulas. 


\section{Appendix B. Log likelihood for the MF12 specification.}

The $P$ dynamics can again be represented as a special case of (1) by using the companion form $F_{t}=\left(F_{t}^{m^{\prime}}, f_{t}^{\ell \prime}\right)^{\prime}, \quad F_{t}^{m}=\left(f_{t}^{m \prime}, \ldots, f_{t-11}^{m \prime}\right)^{\prime}, c=\left(0_{24 \times 1}^{\prime}, c_{\ell}^{\prime}\right)^{\prime}$, and

$$
\begin{gathered}
\rho=\left[\begin{array}{ccccccc}
\rho_{1} & \rho_{2} & \rho_{3} & \cdots & \rho_{11} & \rho_{12} & 0 \\
(2 \times 2) & & & & & & \\
I_{2} & 0 & 0 & \cdots & 0 & 0 & 0 \\
0 & I_{2} & 0 & \cdots & 0 & 0 & 0 \\
\vdots & \vdots & \vdots & \ddots & \vdots & \vdots & \vdots \\
0 & 0 & 0 & \cdots & 0 & 0 & 0 \\
0 & 0 & 0 & \cdots & I_{2} & 0 & 0 \\
0 & 0 & 0 & \cdots & 0 & 0 & \rho_{\ell \ell} \\
& & & & & & (3 \times 3)
\end{array}\right] \\
\Sigma=\left[\begin{array}{cccccc}
\Sigma_{m m} & 0 & \cdots & 0 & 0 \\
(2 \times 2) & & & 0 & 0 \\
0 & 0 & \cdots & 0 & 0 \\
\vdots & \vdots & \ddots & \vdots & \vdots \\
0 & 0 & \cdots & 0 & 0 \\
0 & 0 & \cdots & 0 & \Sigma_{\ell \ell} \\
& & & & (3 \times 3)
\end{array}\right] .
\end{gathered}
$$

Ang and Piazzesi assumed that the risk associated with lagged macro factors is not priced and imposed the restriction in a $\lambda$ representation that the values in (9) are characterized by $\lambda=\left(\lambda_{m}^{\prime}, 0_{22 \times 1}^{\prime}, \lambda_{\ell}^{\prime}\right)^{\prime}$ and

$$
\underset{(27 \times 27)}{\Lambda}=\left[\begin{array}{ccccc}
\Lambda_{m m} & 0 & \cdots & 0 & 0 \\
(2 \times 2) & & & & \\
0 & 0 & \cdots & 0 & 0 \\
\vdots & \vdots & \ddots & \vdots & \vdots \\
0 & 0 & \cdots & 0 & 0 \\
0 & 0 & \cdots & 0 & \Lambda_{\ell \ell} \\
& & & & (3 \times 3)
\end{array}\right]
$$

From $(10)$ and (11) it follows that the parameters in (12) are given by $c^{Q}=\left(c_{m}^{Q \prime}, 0_{22 \times 1}^{\prime}, c_{\ell}^{Q^{\prime}}\right)^{\prime}$ and

$$
\rho^{Q}=\left[\begin{array}{ccccccc}
\rho_{1}^{Q} & \rho_{2} & \rho_{3} & \cdots & \rho_{11} & \rho_{12} & 0 \\
(2 \times 2) & & & & & & \\
I_{2} & 0 & 0 & \cdots & 0 & 0 & 0 \\
0 & I_{2} & 0 & \cdots & 0 & 0 & 0 \\
\vdots & \vdots & \vdots & \ddots & \vdots & \vdots & \vdots \\
0 & 0 & 0 & \cdots & 0 & 0 & 0 \\
0 & 0 & 0 & \cdots & I_{2} & 0 & 0 \\
0 & 0 & 0 & \cdots & 0 & 0 & \rho_{\ell \ell}^{Q} \\
& & & & & & (3 \times 3)
\end{array}\right]
$$


Ang and Piazzesi used $N_{\ell}=3$ and $N_{e}=2$, assuming that the 1-, 12-, and 60-month yields were priced without error, while the 3 - and 36-month yields were priced with error, so that the $B$ matrices can be written in partitioned form as

$$
\left[\begin{array}{ccc}
B_{1 m}^{(0)} & B_{1 m}^{(1)} & B_{1 \ell} \\
(3 \times 2) & (3 \times 22) & (3 \times 3) \\
B_{2 m}^{(0)} & B_{2 m}^{(1)} & B_{2 \ell} \\
(2 \times 2) & (2 \times 22) & (2 \times 3)
\end{array}\right]=\left[\begin{array}{c}
b_{1}^{\prime} \\
b_{12}^{\prime} \\
b_{60}^{\prime} \\
b_{3}^{\prime} \\
b_{36}^{\prime}
\end{array}\right]
$$

where for example $B_{1 m}^{(1)}$ are the coefficients relating the observed yields to 11 lags of the 2 macro factors.

The conditional density for this case is then

$$
\begin{gathered}
f\left(f_{t}^{m}, Y_{t} \mid F_{t-1}^{m}, Y_{t-1}\right)=\frac{1}{|\operatorname{det}(J)|} f\left(f_{t}^{m}, f_{t}^{\ell}, u_{t}^{e} \mid F_{t-1}^{m}, f_{t-1}^{\ell}, u_{t-1}^{e}\right) \\
f\left(f_{t}^{m}, f_{t}^{\ell}, u_{t}^{e} \mid F_{t-1}^{m}, f_{t-1}^{\ell}, u_{t-1}^{e}\right)=f\left(f_{t}^{m} \mid F_{t-1}^{m}\right) f\left(f_{t}^{\ell} \mid f_{t-1}^{\ell}\right) f\left(u_{t}^{e}\right) \\
f\left(f_{t}^{m} \mid F_{t-1}^{m}\right)=\phi\left(f_{t}^{m} ; \rho_{1} f_{t-1}^{m}+\rho_{2} f_{t-2}^{m}+\cdots+\rho_{12} f_{t-12}^{m}, \Sigma_{m m} \Sigma_{m m}^{\prime}\right) \\
f\left(f_{t}^{\ell} \mid f_{t-1}^{\ell}\right)=\phi\left(f_{t}^{\ell} ; \rho_{\ell \ell} f_{t-1}^{\ell}, I_{N_{\ell}}\right) \\
f\left(u_{t}^{e}\right)=\phi\left(u_{t}^{e} ; 0, I_{N_{e}}\right) \\
f_{t}^{\ell}=B_{1 \ell}^{-1}\left(Y_{t}^{1}-A_{1}-\left[\begin{array}{cc}
B_{1 m}^{(0)} & B_{1 m}^{(1)}
\end{array}\right] F_{t}^{m}\right) \\
u_{t}^{e}=\Sigma_{e}^{-1}\left(Y_{t}^{2}-A_{2}-\left[\begin{array}{cc}
B_{2 m}^{(0)} & B_{2 m}^{(1)}
\end{array}\right] F_{t}^{m}-B_{2 \ell} f_{t}^{\ell}\right) \\
J=\left[\begin{array}{cc}
B_{1 \ell} & 0 \\
B_{2 \ell} & \Sigma_{e}
\end{array}\right] .
\end{gathered}
$$

\section{Appendix C. Proof of Proposition 1.}

Write

$$
H=\left[\begin{array}{ll}
u & x \\
v & y
\end{array}\right] .
$$

Since columns of $H$ have unit length, without loss of generality we can write $(u, v)=(\cos \theta, \sin \theta)$ for some $\theta \in[-\pi, \pi]$. The second column of $H$ is also a point on the unit circle, for which orthogonality with the first column also requires it to be located on the line $u x+v y=0$, with the two solutions $x=-v, y=u$ and $x=v, y=-u$. Thus the set of orthogonal $(2 \times 2)$ matrices can be represented as either rotations

$$
H_{1}(\theta)=\left[\begin{array}{cc}
\cos \theta & -\sin \theta \\
\sin \theta & \cos \theta
\end{array}\right]
$$


or reflections

$$
H_{2}(\theta)=\left[\begin{array}{cc}
\cos \theta & \sin \theta \\
\sin \theta & -\cos \theta
\end{array}\right] .
$$

The condition that the $(1,2)$ element of $H_{1}(\theta) \rho H_{1}(\theta)^{\prime}$ be zero requires

$$
\left(\rho_{11}^{Q}-\rho_{22}^{Q}\right) \sin \theta \cos \theta-\rho_{21}^{Q} \sin ^{2} \theta=0 .
$$

One way this could happen is if $\sin \theta=0$. But this would imply either $H_{1}(-\pi / 2)=-I_{2}$, violating the sign requirement $H \delta_{1} \geq 0$, or else the identity transformation $H_{1}(\pi / 2)=I_{2}$. Hence the condition of interest is

$$
\left(\rho_{11}^{Q}-\rho_{22}^{Q}\right) \cos \theta-\rho_{21}^{Q} \sin \theta=0 .
$$

If $\theta_{1}$ satisfies condition (C.3), then one can show

$$
H_{1}\left(\theta_{1}\right) \rho^{Q} H_{1}\left(\theta_{1}\right)^{\prime}=\left[\begin{array}{cc}
\rho_{22}^{Q} & 0 \\
\rho_{21}^{Q} & \rho_{11}^{Q}
\end{array}\right] .
$$

Alternatively for $H_{2}(\theta)$ we have the requirement

$$
\left(\rho_{11}^{Q}-\rho_{22}^{Q}\right) \sin \theta \cos \theta+\rho_{21}^{Q} \sin ^{2} \theta=0
$$

for which the solution $\sin \theta=0$ would violate $H_{2}(\theta) \delta_{1} \geq 0$, leaving the sole condition

$$
\left(\rho_{11}^{Q}-\rho_{22}^{Q}\right) \cos \theta+\rho_{21}^{Q} \sin \theta=0 .
$$

For any $\theta_{2}$ satisfying (C.4),

$$
H_{2}\left(\theta_{2}\right) \rho^{Q} H_{2}\left(\theta_{2}\right)^{\prime}=\left[\begin{array}{cc}
\rho_{22}^{Q} & 0 \\
-\rho_{21}^{Q} & \rho_{11}^{Q}
\end{array}\right] .
$$

Now consider the nonnegativity condition. Since $\cot \theta$ is monotonic on $(0, \pi)$ and repeats the pattern on $(-\pi, 0)$, there are two values $\theta \in[-\pi, \pi]$ satisfying (C.3). We denote the first by $\theta_{1} \in[0, \pi]$, in which case the second is given by $\theta_{1}-\pi$. The two solutions to (C.4) can then be written as $-\theta_{1}$ and $-\theta_{1}+\pi$. We are then looking at 4 possible transformations:

$$
\begin{gathered}
H_{1}\left(\theta_{1}\right) \delta_{1}=\left[\begin{array}{cc}
\cos \theta_{1} & -\sin \theta_{1} \\
\sin \theta_{1} & \cos \theta_{1}
\end{array}\right]\left[\begin{array}{l}
\delta_{11} \\
\delta_{12}
\end{array}\right]=\left[\begin{array}{c}
\delta_{11} \cos \theta_{1}-\delta_{12} \sin \theta_{1} \\
\delta_{11} \sin \theta_{1}+\delta_{12} \cos \theta_{1}
\end{array}\right] \equiv\left[\begin{array}{l}
\delta_{11}^{*} \\
\delta_{12}^{*}
\end{array}\right] \\
H_{1}\left(\theta_{1}-\pi\right) \delta_{1}=\left[\begin{array}{cc}
-\cos \theta_{1} & \sin \theta_{1} \\
-\sin \theta_{1} & -\cos \theta_{1}
\end{array}\right]\left[\begin{array}{l}
\delta_{11} \\
\delta_{12}
\end{array}\right]=\left[\begin{array}{c}
-\delta_{11}^{*} \\
-\delta_{12}^{*}
\end{array}\right] \\
H_{2}\left(-\theta_{1}\right) \delta_{1}=\left[\begin{array}{cc}
\cos \theta_{1} & -\sin \theta_{1} \\
-\sin \theta_{1} & -\cos \theta_{1}
\end{array}\right]\left[\begin{array}{l}
\delta_{11} \\
\delta_{12}
\end{array}\right]=\left[\begin{array}{c}
\delta_{11}^{*} \\
-\delta_{12}^{*}
\end{array}\right] \\
H_{2}\left(-\theta_{1}+\pi\right)=\left[\begin{array}{cc}
-\cos \theta_{1} & \sin \theta_{1} \\
\sin \theta_{1} & \cos \theta_{1}
\end{array}\right]\left[\begin{array}{l}
\delta_{11} \\
\delta_{12}
\end{array}\right]=\left[\begin{array}{c}
-\delta_{11}^{*} \\
\delta_{12}^{*}
\end{array}\right] .
\end{gathered}
$$


Apart from the knife-edge condition $\delta_{11}^{*}=0$ or $\delta_{12}^{*}=0$ (which would require a particular relation between the elements of the original $\rho^{Q}$ and $\delta_{1}$ ), one and only one of the above four vectors would have both elements positive, and this matrix produces $H \rho^{Q} H^{\prime}$ of one of the two specified forms.

For $N_{\ell}>2$, one can construct a family of such orthogonal matrices, for example using a matrix like

$$
H(\theta)=\left[\begin{array}{ccc}
\cos \theta & 0 & -\sin \theta \\
0 & 1 & 0 \\
\sin \theta & 0 & \cos \theta
\end{array}\right]
$$

for $\theta$ satisfying $\rho_{31}^{Q} \sin \theta=\left(\rho_{11}^{Q}-\rho_{33}^{Q}\right) \cos \theta$, which swaps the $(1,1)$ and $(3,3)$ elements of $\rho^{Q}$. Exactly one of the 4 possible matrices performing this swap will preserve positive $H \delta_{1}$. There are $N_{\ell}$ choices for the value one can put into the $(1,1)$ element as a result of such swaps, $N_{\ell}-1$ remaining choices for $\rho_{22}^{Q}$, or a total of $N_{\ell}$ ! permutations.

\section{Appendix D. Proof of Proposition 2.}

Consider first rotations $H_{1}(\theta)$ as specified in (C.1). The $(1,1)$ element of $\Upsilon=H_{1}(\theta) \rho^{Q}\left[H_{1}(\theta)\right]^{\prime}$ is seen to be

$$
h_{1}(\theta)=\rho_{11}^{Q} \cos ^{2} \theta-\left(\rho_{21}^{Q}+\rho_{12}^{Q}\right) \cos \theta \sin \theta+\rho_{22}^{Q} \sin ^{2} \theta .
$$

We claim first that there exists a $\theta \in[0, \pi / 2]$ such that $h_{1}(\theta)$ equals $\left(\rho_{11}^{Q}+\rho_{22}^{Q}\right) / 2$. To see this, note that at $\theta=0$, the value of $h_{1}(\theta)$ is $\rho_{11}^{Q}$, whereas at $\theta=\pi / 2$, it is instead equal to $\rho_{22}^{Q}$. Since $h_{1}(\theta)$ is continuous in $\theta$, there exists a value $\theta_{1}$ such that $h_{1}\left(\theta_{1}\right)$ is exactly halfway between $\rho_{11}^{Q}$ and $\rho_{22}^{Q}$.

Notice next that the eigenvalues of $\Upsilon=H \rho^{Q} H^{\prime}$ are identical to those of $\rho^{Q}$, and hence the trace of $\Upsilon$ (which is the sum of the eigenvalues) is the same as the trace of $\rho^{Q}$ :

$$
\Upsilon_{11}+\Upsilon_{22}=\rho_{11}^{Q}+\rho_{22}^{Q}
$$

Thus since $\Upsilon_{11}=\left(\rho_{11}^{Q}+\rho_{22}^{Q}\right) / 2$, then also $\Upsilon_{22}=\left(\rho_{11}^{Q}+\rho_{22}^{Q}\right) / 2$. Hence $H_{1}\left(\theta_{1}\right) \rho^{Q}\left[H_{1}\left(\theta_{1}\right)\right]^{\prime}$ is of the desired form with elements along the principal diagonal equal to each other. As in the proof of Proposition $1, H_{1}\left(\theta_{1}-\pi\right)$ is the other rotation that works.

Alternatively, $H$ could be a reflection matrix $H_{2}(\theta)$ as in (C.2), for which the $(1,1)$ element of $H_{2}(\theta) \rho^{Q}\left[H_{2}(\theta)\right]^{\prime}$ is found to be:

$$
\rho_{11}^{Q} \cos ^{2} \theta+\left(\rho_{21}^{Q}+\rho_{12}^{Q}\right) \cos \theta \sin \theta+\rho_{22}^{Q} \sin ^{2} \theta
$$

This turns out to equal $\left(\rho_{11}^{Q}+\rho_{22}^{Q}\right) / 2$ at $\theta_{2}=-\theta_{1}$ and $\theta_{2}=-\theta_{1}+\pi$. As in the proof of Proposition 1, in the absence of knfe-edge conditions on $\delta_{1}$, exactly one of the transformations $H_{1}\left(\theta_{1}\right), H_{1}\left(\theta_{1}-\pi\right), H_{2}\left(-\theta_{1}\right), H_{2}\left(-\theta_{1}+\pi\right)$ preserves positivity of $H \delta_{1}$, establishing existence.

For uniqueness, suppose we have found a transformation $H \rho^{Q} H^{\prime}=\Upsilon$ of the desired form. Then any alternative transformation $H^{*} \rho^{Q} H^{* \prime}$ can equivalently be written as $\tilde{H} \Upsilon \tilde{H}^{\prime}$ for $\tilde{H} H=$ $H^{*}$. Hence the result will be established if we can show that the only transformations $\tilde{H} \Upsilon \tilde{H}^{\prime}$ that keep the diagonal elements equal to each other and also satisfy $\tilde{H} \delta_{1} \geq 0$ are the identity and transposition. Since $a=\Upsilon_{11}=\Upsilon_{22}$ and since the transformation preserves eigenvalues, 
we know that if the $(1,1)$ and $(2,2)$ elements of $\tilde{H} \Upsilon \tilde{H}^{\prime}$ are equal to each other, each must again be the value $a$. Thus if $\tilde{H}=H_{1}(\theta)$ for some $\theta$, we require as in (D.1) that

$$
a \cos ^{2} \theta-\left(\Upsilon_{21}+\Upsilon_{12}\right) \cos \theta \sin \theta+a \sin ^{2} \theta=a
$$

which can only be true if

$$
\left(\Upsilon_{21}+\Upsilon_{12}\right) \cos \theta \sin \theta=0
$$

This requires either $\cos \theta=0, \sin \theta=0$, or $\Upsilon_{21}=-\Upsilon_{12}$. For $\cos \theta=0, H_{1}(\theta) \delta_{1}$ would violate the nonnegativity condition, while $\sin \theta=0$ corresponds to $H_{1}(\theta)= \pm I_{2}$. Finally, if $\Upsilon_{21}=-\Upsilon_{12}$, one can verify that $H_{1}(\theta) \Upsilon\left[H_{2}(\theta)\right]^{\prime}=\Upsilon$ for all $\theta$. Alternatively, for reflections applied to a matrix $\Upsilon$ for which $a=\Upsilon_{11}=\Upsilon_{22}$, we see as in (D.2) that $a \cos ^{2} \theta+\left(\Upsilon_{21}+\right.$ $\left.\Upsilon_{12}\right) \cos \theta \sin \theta+a \sin ^{2} \theta=a$, which again can only hold for $\theta$ satisfying (D.3). In this case, $\sin \theta=0$ is ruled out by the constraint $H_{2}(\theta) \delta_{1} \geq 0$, but for $\cos \theta=0$ we have

$$
H_{2}(\pi / 2)=\left[\begin{array}{ll}
0 & 1 \\
1 & 0
\end{array}\right] \quad \text { and } H_{2}(-\pi / 2)=\left[\begin{array}{cc}
0 & -1 \\
-1 & 0
\end{array}\right] \text {. }
$$

Both of these give $H \Upsilon H^{\prime}=\Upsilon^{\prime}$ but only $H_{2}(\pi / 2) \delta_{1}>0$. Finally, when $\Upsilon_{21}=-\Upsilon_{12}$, then $H_{2}(\theta) \Upsilon\left[H_{2}(\theta)\right]^{\prime}=\Upsilon^{\prime}$ for any $\theta$. Thus the only transformation $\tilde{H} \Upsilon \tilde{H}^{\prime}$ that preserves equality of diagonal elements is transposition, as claimed.

\section{Appendix E. Asymptotic standard errors of MLE.}

Here we demonstrate that under the usual regularity conditions,

$$
E\left[\left.\frac{\partial^{2} \mathcal{L}(\pi(\theta) ; Y)}{\partial \theta \partial \theta^{\prime}}\right|_{\theta=\theta_{0}}\right]=-T \Gamma^{\prime} R \Gamma
$$

for

$$
\begin{gathered}
\Gamma=\left[\left.\frac{\partial \pi(\theta)}{\partial \theta^{\prime}}\right|_{\theta=\theta_{0}}\right] \\
R=-T^{-1} E\left[\left.\frac{\partial^{2} \mathcal{L}(\pi ; Y)}{\partial \pi \partial \pi^{\prime}}\right|_{\pi=\pi_{0}}\right] .
\end{gathered}
$$

Note

$$
\frac{\partial \mathcal{L}(\pi(\theta) ; Y)}{\partial \theta^{\prime}}=\left[\begin{array}{lll}
\frac{\partial \mathcal{L}(\pi)}{\partial \pi_{1}} & \cdots & \frac{\partial \mathcal{L}(\pi)}{\partial \pi_{q}}
\end{array}\right]\left[\begin{array}{ccc}
\frac{\partial \pi_{1}}{\partial \theta_{1}} & \cdots & \frac{\partial \pi_{1}}{\partial \theta_{N}} \\
\vdots & \vdots & \vdots \\
\frac{\partial \pi_{q}}{\partial \theta_{1}} & \cdots & \frac{\partial \pi_{q}}{\partial \theta_{N}}
\end{array}\right]
$$




$$
\begin{aligned}
\frac{\partial^{2} \mathcal{L}(\pi(\theta) ; Y)}{\partial \theta_{i} \partial \theta^{\prime}}= & {\left[\begin{array}{lll}
\frac{\partial \pi_{1}}{\partial \theta_{i}} & \cdots & \frac{\partial \pi_{q}}{\partial \theta_{i}}
\end{array}\right]\left[\begin{array}{ccc}
\frac{\partial^{2} \mathcal{L}(\pi)}{\partial \pi_{1} \partial \pi_{1}} & \cdots & \frac{\partial^{2} \mathcal{L}(\pi)}{\partial \pi_{1} \partial \pi_{q}} \\
\vdots & \vdots & \vdots \\
\frac{\partial^{2} \mathcal{L}(\pi)}{\partial \pi_{q} \partial \pi_{1}} & \cdots & \frac{\partial^{2} \mathcal{L}(\pi)}{\partial \pi_{q} \partial \pi_{q}}
\end{array}\right]\left[\begin{array}{ccc}
\frac{\partial \pi_{1}}{\partial \theta_{1}} & \cdots & \frac{\partial \pi_{1}}{\partial \theta_{N}} \\
\vdots & \vdots & \vdots \\
\frac{\partial \pi_{q}}{\partial \theta_{1}} & \cdots & \frac{\partial \pi_{q}}{\partial \theta_{N}}
\end{array}\right] } \\
& +\left[\begin{array}{llll}
\frac{\partial \mathcal{L}(\pi)}{\partial \pi_{1}} & \cdots & \frac{\partial \mathcal{L}(\pi)}{\partial \pi_{q}}
\end{array}\right]\left[\begin{array}{ccc}
\frac{\partial^{2} \pi_{1}}{\partial \theta_{1} \partial \theta_{i}} & \cdots & \frac{\partial^{2} \pi_{1}}{\partial \theta_{N} \partial \theta_{i}} \\
\vdots & \vdots & \vdots \\
\frac{\partial^{2} \pi_{q}}{\partial \theta_{1} \partial \theta_{i}} & \cdots & \frac{\partial^{2} \pi_{q}}{\partial \theta_{N} \partial \theta_{i}}
\end{array}\right] .
\end{aligned}
$$

Evaluate (E.1) at $\theta=\theta_{0}$, take expectations with respect to the distribution of $Y$, and use the fact that $\Gamma$ is not a function of $Y$ :

$$
\begin{aligned}
& E\left[\left.\frac{\partial^{2} \mathcal{L}(\pi(\theta) ; Y)}{\partial \theta_{i} \partial \theta^{\prime}}\right|_{\theta=\theta_{0}}\right]=e_{i}^{\prime} \Gamma^{\prime} E\left[\begin{array}{ccc}
\left.\frac{\partial^{2} \mathcal{L}(\pi)}{\partial \pi_{1} \partial \pi_{1}}\right|_{\pi=\pi_{0}} & \cdots & \left.\frac{\partial^{2} \mathcal{L}(\pi)}{\partial \pi_{1} \partial \pi_{q}}\right|_{\pi=\pi_{0}} \\
\vdots & \vdots & \vdots \\
\left.\frac{\partial^{2} \mathcal{L}(\pi)}{\partial \pi_{q} \partial \pi_{1}}\right|_{\pi=\pi_{0}} & \cdots & \left.\frac{\partial^{2} \mathcal{L}(\pi)}{\partial \pi_{q} \partial \pi_{q}}\right|_{\pi=\pi_{0}}
\end{array}\right] \Gamma \\
& +\left\{E\left[\left.\left.\frac{\partial \mathcal{L}(\pi)}{\partial \pi_{1}}\right|_{\pi=\pi_{0}} \quad \cdots \quad \frac{\partial \mathcal{L}(\pi)}{\partial \pi_{q}}\right|_{\pi=\pi_{0}}\right]\right\}\left[\begin{array}{ccc}
\left.\frac{\partial^{2} \pi_{1}}{\partial \theta_{1} \partial \theta_{i}}\right|_{\theta=\theta_{0}} & \cdots & \left.\frac{\partial^{2} \pi_{1}}{\partial \theta_{N} \partial \theta_{i}}\right|_{\theta=\theta_{0}} \\
\vdots & \vdots & \vdots \\
\left.\frac{\partial^{2} \pi_{q}}{\partial \theta_{1} \partial \theta_{i}}\right|_{\theta=\theta_{0}} & \cdots & \left.\frac{\partial^{2} \pi_{q}}{\partial \theta_{N} \partial \theta_{i}}\right|_{\theta=\theta_{0}}
\end{array}\right] .
\end{aligned}
$$

But the usual regularity conditions imply $E\left\{\partial \mathcal{L}(\pi) /\left.\partial \pi_{j}\right|_{\pi=\pi_{0}}\right\}=0$, so the second term in (E.2) vanishes. Stacking the row vectors represented by the first term into a matrix produces

$$
E\left[\left.\frac{\partial^{2} \mathcal{L}(\pi(\theta) ; Y)}{\partial \theta \partial \theta^{\prime}}\right|_{\theta=\theta_{0}}\right]=\Gamma^{\prime} E\left[\begin{array}{ccc}
\left.\frac{\partial^{2} \mathcal{L}(\pi)}{\partial \pi_{1} \partial \pi_{1}}\right|_{\pi=\pi_{0}} & \cdots & \left.\frac{\partial^{2} \mathcal{L}(\pi)}{\partial \pi_{1} \partial \pi_{q}}\right|_{\pi=\pi_{0}} \\
\vdots & \vdots & \vdots \\
\left.\frac{\partial^{2} \mathcal{L}(\pi)}{\partial \pi_{q} \partial \pi_{1}}\right|_{\pi=\pi_{0}} & \cdots & \left.\frac{\partial^{2} \mathcal{L}(\pi)}{\partial \pi_{q} \partial \pi_{q}}\right|_{\pi=\pi_{0}}
\end{array}\right] \Gamma
$$

as claimed. 


\begin{tabular}{r|rrr|rrr|rrr}
\hline \hline & \multicolumn{3}{|c|}{ True values } & \multicolumn{3}{|c}{ Global maximum } & \multicolumn{3}{c}{ Local 53} \\
\hline$c^{Q}$ & 0.0407 & 0.0135 & 0.5477 & 0.0416 & 0.0085 & 0.5316 & -0.5562 & 0.0204 & 0.0527 \\
$\rho^{Q}$ & 0.9991 & 0 & 0 & 0.9985 & 0 & 0 & 0.9986 & 0 & 0 \\
& 0.0101 & 0.9317 & 0 & 0.0116 & 0.9328 & 0 & 0.0113 & 0.9316 & 0 \\
& 0.0289 & 0.2548 & 0.7062 & 0.0219 & 0.2500 & 0.7202 & 0.0203 & 0.2438 & 0.7352 \\
$\rho$ & 0.9812 & 0.0069 & 0.0607 & 0.9696 & 0.0141 & 0.0671 & 0.9794 & 0.0063 & 0.0840 \\
& -0.0010 & 0.8615 & 0.1049 & -0.0027 & 0.8533 & 0.1175 & -0.0028 & 0.8380 & 0.1267 \\
& 0.0164 & 0.1856 & 0.6867 & 0.0085 & 0.1985 & 0.6993 & 0.0333 & 0.1923 & 0.7202 \\
$\delta_{0}$ & 0.0046 & & & 0.0046 & & & 0.1344 & & \\
$\delta_{1}$ & $1.729 \mathrm{E}-4$ & $1.803 \mathrm{E}-4$ & $4.441 \mathrm{E}-4$ & $1.71 \mathrm{E}-4$ & $1.71 \mathrm{E}-4$ & $4.45 \mathrm{E}-4$ & $1.72 \mathrm{E}-4$ & $1.59 \mathrm{E}-4$ & $4.54 \mathrm{E}-4$ \\
$\Sigma_{e}$ & $9.149 \mathrm{E}-5$ & & & $9.105 \mathrm{E}-5$ & & & $9.110 \mathrm{E}-5$ & & \\
$\operatorname{eig}(\rho)$ & 0.9879 & 0.9341 & 0.6074 & 0.9734 & 0.9448 & 0.6040 & 1.000 & 0.9306 & 0.6070 \\
$\mathrm{LLF}$ & & & & 28110.4 & & & 28096.5 & &
\end{tabular}

Table 1: Parameter values used for simulation and estimates associated with (1) the global maximum and (2) a representative point of local convergence. 


\begin{tabular}{ll|cccccc}
\hline \hline VAR & No. of & $\Sigma_{e}$ & $\rho^{Q}$ & $\delta_{1}$ & $\rho$ & $c^{Q}$ & $\delta_{0}$ \\
parameter & elements & $N_{e}$ & $N_{\ell}\left(N_{\ell}+1\right) / 2$ & $N_{\ell}$ & $N_{\ell}^{2}$ & $N_{\ell}$ & 1 \\
\hline$\Omega_{2}^{*}$ & $N_{e}$ & $\checkmark$ & & & & & \\
$\phi_{21}^{*}$ & $N_{\ell} N_{e}$ & $\checkmark$ & & & & \\
$\Omega_{1}^{*}$ & $N_{\ell}\left(N_{\ell}+1\right) / 2$ & & $\checkmark$ & $\checkmark$ & & & \\
$\phi_{11}^{*}$ & $N_{\ell}^{2}$ & & $\checkmark$ & $\checkmark$ & $\checkmark$ & & \\
$A_{2}^{*}$ & $N_{e}$ & & $\checkmark$ & $\checkmark$ & & $\checkmark$ & $\checkmark$ \\
$A_{1}^{*}$ & $N_{\ell}$ & & $\checkmark$ & $\checkmark$ & $\checkmark$ & $\checkmark$ & $\checkmark$
\end{tabular}

Table 2: Mapping between structural and reduced-form parameters for the latent factor model. 


\begin{tabular}{|c|c|c|c|c|c|c|c|c|c|}
\hline $\begin{array}{l}\text { VAR } \\
\text { parameter }\end{array}$ & $\begin{array}{l}\text { No. of } \\
\text { elements }\end{array}$ & $\begin{array}{lc}\Sigma_{e} & \Sigma_{m m} \\
N_{e} & N_{m}\left(N_{m}+1\right) / 2\end{array}$ & & $\begin{array}{c}\delta_{1} \\
N_{f}\end{array}$ & $\begin{array}{c}\rho_{m \ell} \\
N_{m} N_{\ell}\end{array}$ & $\begin{array}{c}\rho_{m m} \\
N_{m}^{2}\end{array}$ & $\begin{array}{ll}\rho_{\ell \ell} & \rho_{\ell m} \\
N_{\ell}^{2} & N_{\ell} N_{m}\end{array}$ & $\begin{array}{cc}\delta_{0} & c^{Q} \\
1 & N_{m}\end{array}$ & $\begin{array}{c}c_{m} \\
N_{m}\end{array}$ \\
\hline$\Omega_{2}^{*}$ & $N_{e}$ & $\checkmark$ & & & & & & & \\
\hline$\Omega_{m}^{*}$ & $N_{m}\left(N_{m}+1\right) / 2$ & $\checkmark$ & & & & & & & \\
\hline$\psi_{1 m}^{*}$ & $N_{\ell} N_{m}$ & & $\checkmark$ & $\checkmark$ & & & & & \\
\hline$\phi_{2 m}^{*}$ & $N_{e} N_{m}$ & & $\checkmark$ & $\checkmark$ & & & & & \\
\hline$\phi_{21}^{*}$ & $N_{e} N_{\ell}$ & & $\checkmark$ & $\checkmark$ & & & & & \\
\hline$\Omega_{1}^{*}$ & $N_{\ell}\left(N_{\ell}+1\right) / 2$ & & $\checkmark$ & $\checkmark$ & & & & & \\
\hline$\phi_{m 1}^{*}$ & $N_{m} N_{\ell}$ & & $\checkmark$ & $\checkmark$ & $\checkmark$ & & & & \\
\hline$\phi_{m m}^{*}$ & $N_{m}^{2}$ & & $\checkmark$ & $\checkmark$ & $\checkmark$ & $\checkmark$ & & & \\
\hline$\phi_{11}^{*}$ & $N_{\ell}^{2}$ & & $\checkmark$ & $\checkmark$ & & & $\checkmark$ & & \\
\hline$\phi_{1 m}^{*}$ & $N_{\ell} N_{m}$ & & $\checkmark$ & $\checkmark$ & & & $\checkmark$ & & \\
\hline$A_{2}^{*}$ & $N_{e}$ & $\checkmark$ & $\checkmark$ & $\checkmark$ & & & & $\checkmark \checkmark$ & \\
\hline$A_{m}^{*}$ & $N_{m}$ & $\checkmark$ & $\checkmark$ & $\checkmark$ & $\checkmark$ & & & $\checkmark \checkmark$ & $\checkmark$ \\
\hline$A_{1}^{*}$ & $N_{\ell}$ & $\checkmark$ & $\checkmark$ & $\checkmark$ & & & $\checkmark$ & $\checkmark \checkmark$ & \\
\hline
\end{tabular}

Table 3: Mapping between structural and reduced-form parameters for the MF1 model. 


\begin{tabular}{ll|cccccccccc}
\hline \hline VAR & No. of & $\Sigma_{e}$ & $\Sigma_{m m}$ & $\rho_{1, \ldots, 12}$ & $\Lambda_{m m}$ & $\delta_{1 m}$ & $\rho_{\ell \ell}$ & $\Lambda_{\ell \ell}$ & $\delta_{1 \ell}$ & $\delta_{0}$ & $\lambda$ \\
parameter & elements & 2 & 3 & 48 & 4 & 2 & 6 & 9 & 3 & 1 & 5 \\
\hline$\Omega_{2}^{*}$ & 2 & $\checkmark$ & & & & & & & & & \\
$\Omega_{m}^{*}$ & 3 & & $\checkmark$ & & & & & & & & \\
$\phi_{m m}^{*}$ & 48 & & & $\checkmark$ & & & & & & & \\
$\psi_{1 m}^{*}$ & 6 & & & $\checkmark$ & $\checkmark$ & $\checkmark$ & & & & & \\
$\phi_{21}^{*}$ & 6 & & & & & & $\checkmark$ & $\checkmark$ & $\checkmark$ & & \\
$\Omega_{1}^{*}$ & 6 & & & & & & $\checkmark$ & $\checkmark$ & $\checkmark$ & & \\
$\phi_{11}^{*}$ & 9 & & & & & & $\checkmark$ & $\checkmark$ & $\checkmark$ & & \\
$\phi_{2 m}^{*}$ & 48 & & & $\checkmark$ & $\checkmark$ & $\checkmark$ & $\checkmark$ & $\checkmark$ & $\checkmark$ & & \\
$\phi_{1 m}^{*}$ & 72 & & & $\checkmark$ & $\checkmark$ & $\checkmark$ & $\checkmark$ & $\checkmark$ & $\checkmark$ & & \\
$A_{2}^{*}$ & 2 & & $\checkmark$ & $\checkmark$ & $\checkmark$ & $\checkmark$ & $\checkmark$ & $\checkmark$ & $\checkmark$ & $\checkmark$ & $\checkmark$ \\
$A_{1}^{*}$ & 3 & & $\checkmark$ & $\checkmark$ & $\checkmark$ & $\checkmark$ & $\checkmark$ & $\checkmark$ & $\checkmark$ & $\checkmark$ & $\checkmark$
\end{tabular}

Table 4: Mapping between structural and reduced-form parameters for the MF12 model. 


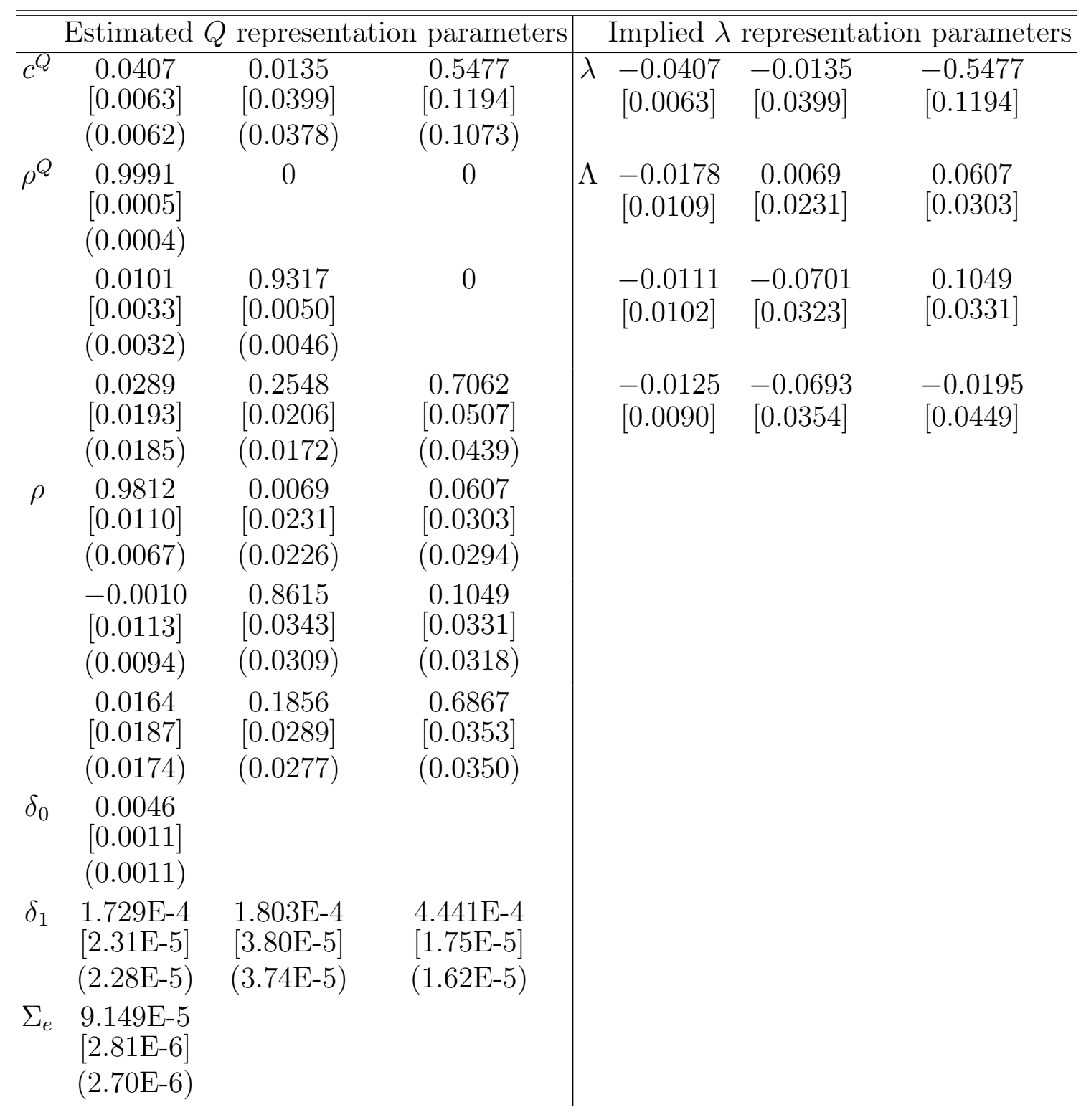

Table 5: FIML estimates with small-sample standard errors (in square brackets) and asymptotic standard errors (in parentheses) for latent factor model fit to Ang and Piazzesi (2003) data set. 


\begin{tabular}{|c|c|c|c|c|c|}
\hline$c^{Q Q}$ & $\begin{array}{c}0.0306 \\
(0.5291)\end{array}$ & $\begin{array}{c}-0.0458 \\
(1.1382)\end{array}$ & "0 & "0 & $\overline{\overline{0}}$ \\
\hline$c$ & $\begin{array}{c}-0.1028 \\
(0.4951)\end{array}$ & $\begin{array}{l}0.2414 \\
(0.4672)\end{array}$ & $\begin{array}{c}-0.9632 \\
(7.2480)\end{array}$ & $\begin{array}{c}-1.5301 \\
(1.4128)\end{array}$ & $\begin{array}{l}2.4063 \\
(4.4009)\end{array}$ \\
\hline \multirow[t]{5}{*}{$\rho^{Q}$} & $\begin{array}{l}0.7725 \\
(0.2895)\end{array}$ & $\begin{array}{c}0.2933 \\
(0.2801)\end{array}$ & $\begin{array}{l}0.0436 \\
(1.0688)\end{array}$ & $\begin{array}{c}-0.2138 \\
(0.1332)\end{array}$ & $\begin{array}{c}-0.3565 \\
(0.3900)\end{array}$ \\
\hline & $\begin{array}{c}-0.3933 \\
(0.3857)\end{array}$ & $\begin{array}{l}1.2411 \\
(0.3706)\end{array}$ & $\begin{array}{c}0.2376 \\
(0.2437)\end{array}$ & $\begin{array}{c}-0.0197 \\
(0.1470)\end{array}$ & $\begin{array}{c}-0.0574 \\
(0.5579)\end{array}$ \\
\hline & $\begin{array}{c}0.2036 \\
(0.3691)\end{array}$ & $\begin{array}{c}-0.2046 \\
(0.3852)\end{array}$ & $\begin{array}{c}0.8579 \\
(0.1435)\end{array}$ & 0 & 0 \\
\hline & $\begin{array}{c}-0.1035 \\
(0.2083)\end{array}$ & $\begin{array}{c}0.1035 \\
(0.2373)\end{array}$ & $\begin{array}{c}-0.0054 \\
(0.5723)\end{array}$ & $\begin{array}{c}0.8826 \\
(0.0672)\end{array}$ & $\begin{array}{c}-0.1926 \\
(0.1464)\end{array}$ \\
\hline & $\begin{array}{l}0.1001 \\
(0.6387)\end{array}$ & $\begin{array}{c}-0.1415 \\
(0.6661)\end{array}$ & $\begin{array}{c}0.0223 \\
(0.1215)\end{array}$ & $\begin{array}{c}0.0303 \\
(0.0810)\end{array}$ & $\begin{array}{l}0.8826 \\
(0.0672)\end{array}$ \\
\hline \multirow[t]{5}{*}{$\rho$} & $\begin{array}{l}0.9461 \\
(0.0325)\end{array}$ & $\begin{array}{l}0.2203 \\
(0.0508)\end{array}$ & $\begin{array}{c}-0.0428 \\
(0.2005)\end{array}$ & $\begin{array}{c}-0.0210 \\
(0.0456)\end{array}$ & $\begin{array}{l}0.0639 \\
(0.1531)\end{array}$ \\
\hline & $\begin{array}{c}0.0002 \\
(0.0310)\end{array}$ & $\begin{array}{l}0.8735 \\
(0.0487)\end{array}$ & $\begin{array}{c}-0.0435 \\
(0.1618)\end{array}$ & $\begin{array}{c}-0.0233 \\
(0.0538)\end{array}$ & $\begin{array}{c}-0.0517 \\
(0.1555)\end{array}$ \\
\hline & $\begin{array}{c}0.0932 \\
(0.3903)\end{array}$ & $\begin{array}{c}0.1683 \\
(0.1686)\end{array}$ & $\begin{array}{l}0.8203 \\
(0.6723)\end{array}$ & $\begin{array}{c}-0.0844 \\
(0.2453)\end{array}$ & $\begin{array}{l}0.1378 \\
(1.0303)\end{array}$ \\
\hline & $\begin{array}{c}-0.0827 \\
(0.1190)\end{array}$ & $\begin{array}{c}0.0852 \\
(0.1295)\end{array}$ & $\begin{array}{c}-0.1110 \\
(0.3430)\end{array}$ & $\begin{array}{l}0.8715 \\
(0.1127)\end{array}$ & $\begin{array}{c}0.0978 \\
(0.2066)\end{array}$ \\
\hline & $\begin{array}{l}0.1220 \\
(0.2649)\end{array}$ & $\begin{array}{c}0.0449 \\
(0.5693)\end{array}$ & $\begin{array}{l}0.0756 \\
(1.0167)\end{array}$ & $\begin{array}{c}0.0555 \\
(0.1468)\end{array}$ & $\begin{array}{l}0.4728 \\
(0.7418)\end{array}$ \\
\hline$\delta_{0}$ & $\begin{array}{c}-0.0082 \\
(0.0062)\end{array}$ & & & & \\
\hline$\delta_{1}$ & $\begin{array}{c}6.86 \mathrm{E}-4 \\
(2.88 \mathrm{E}-4)\end{array}$ & $\begin{array}{l}1.02 \mathrm{E}-3 \\
(3.03 \mathrm{E}-4)\end{array}$ & $\begin{array}{c}2.03 \mathrm{E}-3 \\
(2.35 \mathrm{E}-3)\end{array}$ & $\frac{1.92 \mathrm{E}-4}{(1.33 \mathrm{E}-3)}$ & $\underset{(6.31 \mathrm{E}-3)}{7.67 \mathrm{E}-4}$ \\
\hline$\Sigma_{e}$ & $\begin{array}{c}2.02 \mathrm{E}-4 \\
(1.29 \mathrm{E}-5)\end{array}$ & $\frac{1.87 \mathrm{E}-4}{(1.19 \mathrm{E}-5)}$ & $\frac{1.09 \mathrm{E}-4}{(6.97 \mathrm{E}-6)}$ & & \\
\hline \multirow[t]{2}{*}{$\Sigma_{m m}$} & $\begin{array}{l}0.6996 \\
(0.0448)\end{array}$ & 0 & & & \\
\hline & $\begin{array}{l}0.1174 \\
(0.0604)\end{array}$ & $\begin{array}{l}0.6617 \\
(0.0424)\end{array}$ & & & \\
\hline
\end{tabular}

Table 6: FIML estimates and asymptotic standard errors for the MF1 model. 


\begin{tabular}{|c|c|c|c|c|c|c|c|c|c|}
\hline & \multicolumn{3}{|c|}{$\bar{~} \overline{\text { Global }}$} & \multicolumn{3}{|c|}{ 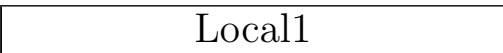 } & \multicolumn{3}{|c|}{ 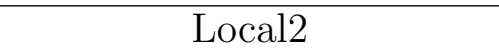 } \\
\hline \multirow[t]{3}{*}{$\rho_{\ell \ell}$} & 0.9921 & 0 & 0 & 0.9918 & 0 & 0 & 0.9920 & 0 & 0 \\
\hline & 0 & 0.9462 & 0 & 0 & 0.9412 & 0 & 0 & 0.9437 & 0 \\
\hline & 0 & -0.0034 & 0.9021 & 0 & -0.0095 & 0.7712 & 0 & -0.0032 & 0.9401 \\
\hline$\delta_{1 \ell}$ & $1.11 \mathrm{E}-04$ & $4.27 \mathrm{E}-04$ & $1.98 \mathrm{E}-04$ & 1.09E-04 & 4.30E-04 & $1.92 \mathrm{E}-04$ & $1.22 \mathrm{E}-04$ & $4.26 \mathrm{E}-04$ & $1.92 \mathrm{E}-04$ \\
\hline$\lambda_{\ell}$ & -0.0409 & 0 & 0 & -0.0441 & 0 & 0 & -0.0388 & 0 & 0 \\
\hline \multirow[t]{2}{*}{$\Lambda_{m m}$} & 2.8783 & 0.4303 & & -0.3430 & 0.1474 & & 1.5633 & 0.1341 & \\
\hline & -6.1474 & -0.8744 & & 1.7675 & -0.0607 & & 16.0624 & 7.4290 & \\
\hline \multirow[t]{3}{*}{$\Lambda_{\ell \ell}$} & -0.0048 & 0 & 0 & -0.0045 & 0 & 0 & -0.0056 & 0 & 0 \\
\hline & -0.0445 & 0 & 0.2910 & -0.0474 & 0 & 0.2881 & -0.0423 & 0 & 0.3000 \\
\hline & -0.0322 & 0 & 0.3687 & -0.0331 & 0 & 0.2110 & -0.0299 & 0 & 0.4120 \\
\hline$\chi^{2}$ & 462.15 & & & 530.69 & & & 503.10 & & \\
\hline LLF & 20703 & & & 20668 & & & 20679 & & \\
\hline Frequency & 14 & & & 84 & & & 2 & & \\
\hline
\end{tabular}

Table 7: Three local minima for the chi-square objective function for the restricted MF12 specification. 